
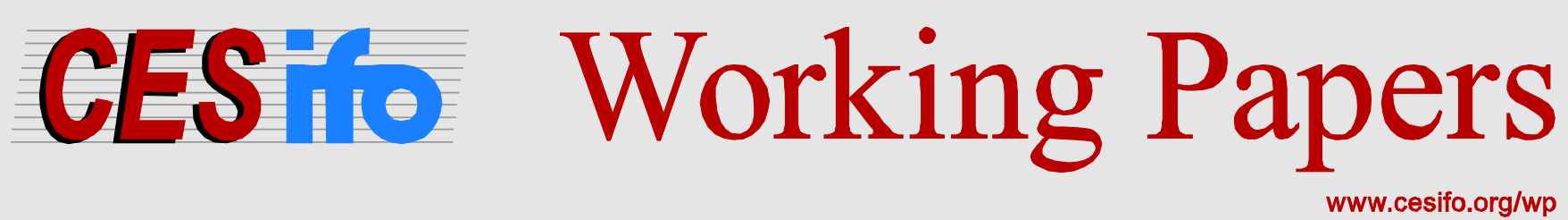

\title{
Individual Heterogeneity, Nonlinear Budget Sets, and Taxable Income
}

\author{
Soren Blomquist \\ Anil Kumar \\ Che-Yuan Liang \\ Whitney K. Newey
}

CESIFO WORKING PAPER NO. 5320

CATEgORY 1: PUBlic FinANCE

APRIL 2015

An electronic version of the paper may be downloaded

- from the SSRN website:

- from the RePEc website:

- from the CESifo website:

WwW.SSRN.com

www.RePEc.org

www.CESifo-group.org/wp 


\title{
Individual Heterogeneity, Nonlinear Budget Sets, and Taxable Income
}

\begin{abstract}
Many studies have estimated the effect of taxes on taxable income. To account for nonlinear taxes these studies either use instrumental variables approaches that are not fully consistent, or impose strong functional form assumptions. None allow for general heterogeneity in preferences. In this paper we derive the expected value and distribution of taxable income, conditional on a nonlinear budget set, allowing general heterogeneity and optimization error in taxable income. We find an important dimension reduction and use that to develop nonparametric estimation methods. We show how to nonparametrically estimate the expected value of taxable income imposing all the restrictions of utility maximization and allowing for measurement errors. We characterize what can be learned nonparametrically from kinks about compensated tax effects. We apply our results to Swedish data and estimate for prime age males a significant net of tax elasticity of 0.21 and a significant nonlabor income effect of about -1 . The income effect is substantially larger in magnitude than found in other taxable income studies.
\end{abstract}

JEL-Code: C140, C240, H300, H310, J220.

Keywords: nonlinear budget sets, nonparametric estimation, heterogeneous preferences, taxable income, revealed stochastic preference.

\author{
Soren Blomquist \\ Uppsala Center for Fiscal Studies \\ Department of Economics \\ Uppsala University / Sweden \\ soren.blomquist@nek.uu.se \\ Che-Yuan Liang \\ Uppsala Center for Fiscal Studies \\ Department of Economics \\ Uppsala University / Sweden \\ che-yuan.liang@nek.uu.se
}

\author{
Anil Kumar \\ Federal Reserve Bank of Dallas \\ Dallas / Texas / USA \\ anil.kumar@dal.frb.org
}

\author{
Whitney K. Newey \\ Department of Economics \\ M.I.T. \\ Cambridge MA 02139 / USA \\ wnewey@mit.edu
}




\section{Introduction}

Behavioral responses to tax changes are of great policy interest. In the past much of this interest was focused on hours of work, and the central question was how labor supply responds to tax reform. In a set of influential papers, Feldstein $(1995,1999)$ emphasized that traditional measures of deadweight loss based just on labor supply are biased downward as they ignore many other important behavioral responses like work effort, job location, tax avoidance and evasion. Inspired by Feldstein's work, which showed that the taxable income elasticity is sufficient for estimating the marginal deadweight loss from taxes, a large number of studies have produced a wide range of estimates. ${ }^{1}$

Although the conventional estimates of the taxable income elasticity provide information on how taxable income reacts to a marginal change in a linear budget constraint, they are less useful for estimating the effect of tax reforms on taxable income. In a real world of nonlinear tax systems with kinks in individuals' budget constraints, tax reforms often result in changes in kink points as well as marginal tax rates for various brackets. There has been extensive research on estimating the effect of such complicated changes in the tax systems on labor supply using parametric structural models with piecewise linear budget sets, often estimated by maximum likelihood methods. More recent labor supply studies have estimated a utility function which can be used to predict the effect of taxes in the presence of piecewise linear budget sets. These studies focusing on labor supply, however, not only ignore other margins of behavioral responses to taxation but also rely on strong distributional and functional form assumptions when they use parametric models.

We nonparametrically identify and estimate the expected value of taxable income conditional on nonlinear budget sets while allowing for general heterogeneity. The heterogenous preferences are assumed to be strictly convex and statistically independent of the budget set, but are otherwise unrestricted. We also allow for optimization errors in estimation of the expected value. We find that for a piecewise linear tax schedule the expected value can be estimated by a low dimensional nonparametric regression. One form of this regression is exactly analogous to the conditional mean of labor supply from Blomquist and Newey (2002, BN henceforth), which was derived when preference heterogeneity can be represented by a scalar. Consequently, it turns out that the labor supply results of BN are valid under general preference heterogeneity. However the $\mathrm{BN}$ form did not impose all the restrictions imposed by utility maximization and we show how to do this.

\footnotetext{
${ }^{1}$ The estimates range from -1.3 (Goolsbee, 1999) to 3 (Feldstein, 1995) with more recent studies closer to 0.5 (Saez, 2003; Gruber and Saez, 2002; Kopczuk, 2005, Giertz, 2007). Blomquist and Selin, 2010 find an elasticity of about .2; See Saez, Slemrod and Giertz (2012) for a comprehensive review of the literature.
} 
We derive the distribution of taxable income at points where the budget frontier is concave over an open interval. We analyze kinks, showing how kinks of any size depend nonparametrically on the density of taxable income as well as average compensated effects for individuals at the kink. We also find that the conditional distribution of taxable income given the budget set is the same as for a linear budget set with the net of tax rate equal to the slope from the right of the budget set and income equal to virtual income. This finding dramatically reduces the dimension of the nonparametric estimation problem, because it shows that the conditional distribution is only two dimensional rather than depending on the whole budget set. Also, we find that varying convex budget sets provides the same information about preferences as varying linear budget sets.

The model here is like the revealed stochastic preference model of McFadden (2005) for continuous outcomes in having general heterogenous preferences and budget sets that are statistically independent of preferences. We differ in considering only the two good case with strictly convex preferences. We find that for two goods, smooth strictly convex preferences, and convex budget sets, necessary and sufficient conditions for utility maximization are that the CDF given a linear budget set satisfies a Slutzky property.

In independent work Manski (2013) considered the identification of general preferences when labor supply is restricted to a finite choice set, with the goal of evaluating tax policy. He found that nonidentification of preferences makes tax policy evaluation difficult. This paper and BN have a different conclusion than Manski (2013), showing that for certain objects of interest, like the expected value of labor supply or taxable income, tax policy can be evaluated via nonparametric regression. The difference is that we integrate over the preference distribution rather than trying to identify it. This allows nonparametric evaluation of certain tax policies, as was done in the prior work of $\mathrm{BN}$ and as is done here. Importantly, $\mathrm{BN}$ and we also allow for a source of variation in the outcome other than preference variation (e.g. measurement error), which has long been thought to be important for labor supply and taxable income; see Burtless and Hausman (1978). Such a specification is not based on utility maximization, unlike Chetty (2012), but does allow for low probabilities for kinks, as is often found in data.

Keane and Moffitt (1998) and Blundell and Shephard (2012) have considered labor supply when hours are restricted to a finite set. Our taxable income setup could accommodate such constraints, though we do not do this for simplicity. As long as the grid of possible labor supply values was rich enough the expected value of taxable income that we derive would be approximately correct. It appears to be harder to incorporate the bilateral contracting framework of Blundell and Shephard (2012).

To evaluate the effect of taxes on taxable income we focus on elasticities that apply to 
changes in nonlinear tax systems. Real-world tax systems are non-linear, and it is variations in non-linear tax systems that we observe. Therefore, it is easiest to nonparametrically identify elasticities relevant for changes in nonlinear tax systems. BN did show that with labor supply it may be possible to identify labor supply elasticities for changes in linear budget sets, but for taxable income we find that the conditions for identifying average elasticities for a linear budget set are very stringent and not likely to be satisfied in applications. Here we propose effects defined by an upward shift of the non-linear budget constraint, in either slope or intercept. These effects are relevant for changes in non-linear budget constraints. We find that these can be estimated with high accuracy in our application.

In the taxable income setting it is important to allow for productivity growth. To nonparametrically separate out the effect of exogenous productivity growth from changes in taxable income that are due to changes in individual behavior is one of the hardest problems in the taxable income literature. We give a way to do this and show how it matters for the results.

Our application is to Swedish data from 1993-2008 with third party reported taxable labor income. This means that the variation in the taxable income that we observe for Sweden is mainly driven by variations in effort broadly defined and variations in hours of work and not by variations in tax evasion. ${ }^{2}$ We estimate a statistically significant tax elasticity of 0.21 and a significant income effect of -1 . This income effect is signicantly larger than in many taxable income studies, many of which find a small effect or assume no effect.

The rest of our paper is organized as follows. Section 2 reviews the taxable income literature. Section 3 lays out a model of individual behavior where there are more decision margins than hours of work. Section 4 derives the distribution of taxable income for nonlinear budget sets. Section 5 analyzes kinks. Section 6 derives the expected value of taxable income and shows how to approximate it in a way that imposes utility maximization. Section 7 describes the policy effects we consider and how we allow for productivity growth. Section 8 explains how these results can be empirically implemented. In Section 9 we describe the Swedish data we use and present our estimates. Section 10 concludes.

\section{Previous literature}

Lindsey (1987) used 1981 ERTA as a natural experiment to estimate a taxable income elasticity of about 1.6 using repeated cross sections from 1980-1984. In his influential paper that brought the taxable income elasticity to the center stage of research on behavioral effects of taxation, Feldstein (1995) used a panel of NBER tax returns and variation from TRA 1986 to estimate

\footnotetext{
${ }^{2}$ Kleven et al. (2011) find that the tax evasion rate is close to zero for income subject to third-party reporting.
} 
elasticity greater than 1 and even higher for high-income individuals for a sample of married individuals with income over $\$ 30,000$. Navratil (1995) also used the 1980-1983 waves of NBER tax panel and using variation from 1981 ERTA on a sample of married people with income more than $\$ 25,000$ he estimated an elasticity of 0.8. Feldstein and Feenberg (1995) used OBRA 1993 as a source of identifying variation and used IRS data from 1992 and 1993 and estimated an elasticity of 1 .

Other papers have found much lower taxable income elasticities. Auten and Caroll (1999) used treasury tax panel from 1985 and 1989, i.e., before and after TRA 1986 to find an elasticity of 0.5. They restricted their sample to individuals earning more than $\$ 15,000$. Sammartino and Weiner (1997) also used treasury tax panel from 1991 and 1994 and variation from OBRA 1993 to estimate zero taxable income elasticity. Goolsbee (1999) used a panel of high-income corporate executives with earnings higher than $\$ 150000$ before and after OBRA 1993. His estimate of the elasticity was close to 0.3 in the long run but close to 1 in the short run. Carroll (1998) also used the treasury tax panel from 1985 to 1989 and found an elasticity of 0.5. Goolsbee (1999) used a long data set from 1922-1989 and used multiple tax reforms as source of identification to find a taxable elasticity ranging from -1.3 to 2 depending on the tax reform.

Moffitt and Wilhelm (2000) used the SCF waves of 1983 and 1989 and exploited TRA 1986 to estimate a much larger elasticity of 2. Gruber and Saez (2002) used alternative definitions of taxable income and used variation from ERTA 1981 and TRA 1986 using the Continuous Work History Files from 1979-1990. Their elasticity estimates were in the range of 0.12-0.4. However, for high-income individuals the elasticity was 0.57 compared with 0.18 for the lower-income individuals. Sillamaa and Veall (2000) used Canadian data from 1986-1989 and identified the taxable income elasticity using Tax Reform Act of 1988. They found taxable income elasticity ranging from 0.14-1.30.

Saez (2003) used the University of Michigan tax panel from 1979-1981 and used the "bracketcreep" due to high inflation to compare income changes of those at the top of the bracket who experienced a change in their marginal tax rate as they crept into an upper bracket to those at the bottom of the tax bracket whose marginal tax rates remained relatively unchanged. Since the two groups are very close in their incomes, these estimates are robust to biases due to increasing income inequality. He estimated an elasticity of 0.4 using taxable income as the definition of income. However, the estimated elasticity was zero once the definition was changed to wage income.

More recent studies have also estimated low taxable income elasticities. Kopczuk (2005) used the University of Michigan tax panel to yield an estimate of -0.2-0.57. More recently Eissa and Giertz (2006) used the Treasury tax panel from 1992-2003 and data from executive 
compensation. They used variation from multiple tax reforms during this period -TRA 1986, OBRA and EGTRRA on a sample of executives and top 1 percent of the tax panel. Their elasticity estimates were small for the long run (0.19), but 0.82 for the short run. Using data from SIPP and the NBER tax panel, Looney and Singhal (2006) also estimate a somewhat larger elasticity of 0.75. More recently Giertz (2007) used Continuous Work History Survey data from 1979 to 2001 and using methods similar to Gruber and Saez (2002) estimated taxable income elasticity of 0.40 for the 1980 s and 0.26 for the 1990s. Using a broader definition of income, the elasticities were 0.21 for the 80s and 0.13 for the 90s. Blomquist and Selin (2010) used Swedish Level of Living Survey combined with register data to estimate an elasticity for taxable income of 0.19-0.21 for men and 0.96-1.44 for females. Using the University of Michigan Tax Panel from 1979-1990 and instrumental variable methods, Weber (2014) found a taxable income elasticity between 0.86 and 1.36 in different specifications.

\section{The Model}

Feldstein (1995) argued that individuals have more margins than hours of work to respond to changes in the tax. For example, they could exert more effort on the present job, switch to a better paid job that requires more effort, or could move geographically to a better-paid job. The choice of compensation mix (cash versus fringe benefits) and tax avoidance/evasion are still other margins. Our data is such that we do not need to worry about tax evasion but allowing for an effort margin seems useful and is important for accounting correctly for productivity growth over time.

To describe the model let $c$ denote consumption, $e$ effort, and $h$ hours of work. Also let $R$ denote nonlabor income and for a linear tax let $\tau$ denote the tax rate and $\rho=1-\tau$ the net of tax rate for income. We let the wage be $w(e)$ for effort level $e$. Let $u(c, e, h)$ denote an individual's utility function, assumed to be strictly quasi-concave, increasing in $c$ and decreasing in $e$ and $h$. The individual choice problem is

$$
\operatorname{Max}_{c, e, h} u(c, e, h) \quad \text { s.t. } \quad c=w(e) h \rho+R, c \geq 0, e \geq 0, h \geq 0 .
$$

This problem can be reformulated as a choice of consumption and taxable income $y=w(e) h$. Since $w(e)=y / h$, if the wage function $w(e)$ is one-to-one then inverting gives $e=w^{-1}(y / h)$. Noting that only $y$ enters the constraint we can concentrate $h$ out of the choice problem by choosing $h$ to maximize $u\left(c, w^{-1}(y / h), h\right)$ and then maximizing over $c$ and $y$. Letting $U(c, y)=$ $\max _{h} u\left(c, w^{-1}(y / h), h\right)$ be a concentrated utility function, the choice of $c$ and $y$ is obtained by solving

$$
\operatorname{Max}_{c, y} U(c, y) \quad \text { s.t. } \quad c=y \rho+R, c \geq 0, y \geq 0 .
$$


The solution gives taxable income $y(\rho, R)$ as a function of the net of tax rate $\rho$ and nonlabor income $R$.

In the taxable income literature one usually starts with individual choice of consumption $c$ and taxable income $y$ as given by equation (3.2). We will also adopt this approach for much of the paper. We do return to the original effort specification when we incorporate productivity changes. We do this because productivity affects wages as a function of effort. Also, through much of the paper we will assume that $U(c, y)$ is strictly quasi-concave. This condition is not equivalent to $u(c, e, h)$ being strictly quasi-concave but instead corresponds to an additional restriction on $u(c, e, h)$. Nevertheless we will assume strict quasi-concavity of $U(c, y)$ throughout consistent with the focus on taxable income.

We allow for general heterogeneity that affects both preferences and wages. Let $\eta$ denote a vector valued random variable of any dimension that represents an individual. We specify the utility function of an individual as $u(c, e, h, \eta)$ and the wage rate as $w=g(e, \eta)$. We impose no restriction on how $\eta$ enters the utility or wage function, thus allowing for distinct heterogeneity in both preferences and the wage function (e.g. ability), with different components of $\eta$ entering $u$ and $w(e)$. The individual's optimization problem for a linear budget set is now to maximize $u(c, e, h, \eta)$ subject to $c=w(e, \eta) h \rho+R$. As before we concentrate out hours using $U(c, y, \eta)=\max _{h} u\left(c, w^{-1}(y / h, \eta), h, \eta\right)$. The choice of taxable labor income $y(\rho, R, \eta)$ for an individual $\eta$ for a linear budget set is then given by

$$
y(\rho, R, \eta)=\arg \max _{c, y} U(c, y, \eta) \text { s.t. } c=y \rho+R, c \geq 0, y \geq 0 .
$$

This is the same choice problem as before except that the concentrated utility function $U$ now depends on $\eta$, and hence so does the taxable income function $y(\rho, R, \eta)$.

This specification allows for preferences to vary across individuals in essentially any way at all. For example income and level effects can vary separately (as in Burtless and Hausman, 1978) and the number of types is not restricted to be finite (unlike Manski, 2013). We do need to restrict $\eta$ and $U(c, y, \eta)$ so that probability statements can be made but these are technical side conditions that do not affect our interpretation of $\eta$ as representing general heterogeneity and are reserved for the Appendix. Here we make the following Assumption about $U(c, y, \eta)$ and $y(\rho, R, \eta)$ :

Assumption 1: For each $\eta, U(c, y, \eta)$ is continuous in $(c, y)$, increasing in $c$, decreasing in $y$, and strictly quasi-concave in $(c, y)$. Also $y(\rho, R, \eta)<\infty$ and $y(\rho, R, \eta)$ is continuously differentiable in $\rho, R>0$.

The strict quasi concavity of $U(c, y, \eta)$ is essentially equivalent to uniqueness of $y(\rho, R, \eta)$. 
The continuity and monotonicity conditions are standard in the taxable income literature. Also, we will use continuous differentiability for some of the results to follow.

The analysis to follow will focus on the CDF of taxable income for a fixed budget set as $\eta$ varies. For a linear budget set this $\operatorname{CDF}$ is that of $y(\rho, R, \eta)$ for fixed $\rho$ and $R$. Let $G$ denote the distribution of $\eta$. The taxable income $\operatorname{CDF} F(y \mid \rho, R)$ for a linear budget set is given by

$$
F(y \mid \rho, R)=\int 1(y(\rho, R, \eta) \leq y) G(d \eta)
$$

This CDF plays a pivotal role in the analysis to follow.

The model we are analyzing is a random utility model (RUM) of the kind considered by McFadden (2005) for continuous choice (see also McFadden and Richter, 1991, for discrete choice). The model here specializes the RUM to $U(c, y, \eta)$ that are strictly quasi-concave and $y(\rho, R, \eta)$ that is smooth in $\rho$ and $R$. Single valued, smooth demand specifications are often used in applications. In particular, smoothness has often proven useful in applications of nonparametric models and it will here.

McFadden (2005) derived restrictions on $F(y \mid \rho, R)$ that are necessary and sufficient for a RUM. With choice over two dimensions ( $c$ and $y$ ) there is a simple, alternative characterization of the RUM. The characterization is that the CDF satisfy a Slutzky like condition, referred to henceforth as the Slutzky condition. The following result holds under technical conditions that are given in Assumption A2 of the Appendix. Let $F_{\rho}(y \mid \rho, R)=\partial F(y \mid \rho, R) / \partial \rho$ and $F_{R}(y \mid \rho, R)=$ $\partial F(y \mid \rho, R) / \partial R$ when these partial derivatives exist.

Theorem 1: If Assumptions 1, A1, and A2 are satisfied then $F(y \mid \rho, R)$ is continuously differentiable in $\rho$ and $R$ and

$$
F_{\rho}(y \mid \rho, R)-y F_{R}(y \mid \rho, R) \leq 0 .
$$

Also, if for all $\rho, R>0, F(y \mid \rho, R)$ is continuously differentiable in $y, \rho, R$, the support of $F(y \mid \rho, R)$ is $\left[y_{\ell}, y_{u}\right], \partial F(y \mid \rho, R) / \partial y>0$ on $\left(y_{\ell}, y_{u}\right)$, and equation (3.4) is satisfied then there is a RUM satisfying Assumption 1.

In this sense, for two goods and single valued smooth demands, the revealed stochastic preference conditions are that the CDF satisfies the Slutzky condition. This result will be used in the analysis to follow and is of interest in its own right. Dette, Hoderlein, and Neumeyer (2011) showed that each quantile of $y(\rho, R, \eta)$ satisfies the Slutzky condition for demand functions under conditions similar to those of Assumption A2. Hausman and Newey (2014) observed that when a quantile function satisfies the Slutzky condition there is always a demand model with that quantile function. Theorem 1 is essentially those results combined with the inverse 
function theorem, that implies that the CDF satisfies the Slutzky condition if and only if the quantile satisfies the Slutzky condition.

\section{Nonlinear Budget Sets}

In practice tax rates vary with income so that the budget frontier is nonlinear. To describe choice in this setting let $B(y)$ denote the maximum obtainable consumption for income $y$ allowed by a tax schedule, that we will refer to as the budget frontier. The set of points $\{(y, B(y)): y \geq 0\}$ will be the frontier of the budget set. Under the monotonicity condition of Assumption 1 that utility is strictly increasing in consumption, the choice $y(B, \eta)$ of taxable income by individual $\eta$ will lie on the budget frontier. This choice is given by

$$
y(B, \eta)=\operatorname{argmax}_{y} U(B(y), y, \eta)
$$

When the budget frontier $B$ is concave the choice $y(B, \eta)$ will be unique by strict quasiconcavity of preferences. In general, when $B$ is not concave the choice $y(B, \eta)$ could be a set. Here we will assume the set valued choices occur with probability zero in the distribution of $\eta$ and so ignore them.

In practice most tax systems have a finite number of rates that change at certain income values. In such cases the budget frontier is piecewise linear. A continuous, piecewise-linear budget set with $J$ segments, indexed by $j$, can be described by a vector $\left(\rho_{1}, \ldots, \rho_{J}, R_{1}, \ldots, R_{J}\right)$ of net-of-tax rates $\rho_{j}$ (slopes) and virtual incomes $R_{j}$ (intercepts). It will have kink points $\ell_{0}=0, \ell_{J}=\infty$, and $\ell_{j}=\left(R_{j+1}-R_{j}\right) /\left(\rho_{j}-\rho_{j+1}\right),(1 \leq j \leq J-1)$. The budget frontier will be

$$
B(y)=\sum_{j=1}^{J} 1\left(\ell_{j-1} \leq y<\ell_{j}\right)\left(R_{j}+\rho_{j} y\right) .
$$

In what follows we will also give some results for the case where budget sets need not be piecewise linear.

In general the CDF of the taxable income $y(B, \eta)$ will depend on the entire frontier function $B$. An important simplification occurs around points where $B(y)$ is concave, i.e. where the marginal tax rate is increasing. Let $\overline{\mathcal{B}}$ denote the convex hull of the budget set and $\bar{B}(y)=$ $\max _{(c, y) \in \overline{\mathcal{B}}} c$ denote the corresponding budget frontier. Note that by standard convex analysis results $\bar{B}(y)$ will be a concave function. Let

$$
\rho(y)=\lim _{z \downarrow y}[\bar{B}(z)-\bar{B}(y)] /(z-y), R(y)=\bar{B}(y)-\rho(y) y,
$$

denote the slope from the right $\rho(y)$ of $\bar{B}(y)$ and $R(y)$ the corresponding virtual income, where the limit $\rho(y)$ exists by Rockafellar (1970, pp. 214-215). Also let $F(y \mid B)=\int 1(y(B, \eta) \leq$ $y) G(d \eta)$ denote the CDF of taxable income for a budget frontier $B$. 
Theorem 2: If Assumptions 1 and A1 are satisfied then for all $y$ such that there is $\Delta>0$ with $\bar{B}(z)=B(z)$ for $z \in[y, y+\Delta]$ we have $F(y \mid B)=F(y \mid \rho(y), R(y))$.

Here we find that the CDF is that of a linear budget set at the right slope $\rho(y)$ and corresponding virtual income $R(y)$ at any value $y$ where the frontier coincides with the frontier of the convex hull on a neighborhood to the right of $y$. The slope from the right $\rho(y)$ and the neighborhood to the right of $y$ appear here because of the weak inequality in the definition of the CDF.

This result is an important dimension reduction in the way the CDF depends on the budget set. In principle $F(y \mid B)$ can depend on the entire frontier $B$, an infinite dimensional object. When the frontier is locally concave (to the right of $y$ ) the CDF depends only on the slope $\rho(y)$ and virtual income $R(y)$ instead of the entire budget set. Furthermore, the CDF is that for a linear budget set. This result has a number of useful implications that are discussed in the rest of the paper. For example, this dimension reduction makes it possible to nonparametrically estimate how the expected value of taxable income varies with convex budget sets, as we do in the application below.

In many applications nonconvexities occur only at small values of income. Theorem 2 could be used to nonparametrically quantify how the CDF depends on budget set at higher values of $y$ where the conditions of Theorem 2 are satisfied. For example, one could nonparametrically estimate the revenue effect of changing taxes on higher income earners. Such an object would be of interest because most of the revenue often comes from those paying higher taxes. We leave this use of Theorem 2 to future work.

Theorem 2 implies a revealed stochastic preference result for convex budget sets. As shown by Theorem 1, for linear budget sets and preference satisfying the conditions of Assumptions 1 and A1, a necessary and sufficient condition for a RUM is that the CDF satisfy the Slutzky condition. An implication of Theorem 2 is that this result is also true for convex budget sets. The CDF of taxable income for convex budget sets is consistent with a RUM if and only if the CDF satisfies the Slutzky condition for linear budget sets.

Theorem 2 can also be used to derive identification results for the CDF and conditional expectation of taxable income for a linear budget set. Let $\mathcal{B}$ denote a set of budget frontiers and $X(y)=\{(\rho(y), R(y)): B \in \mathcal{B}\}$. Then $F(y \mid \rho, R)$ is identified for $(\rho, R) \in X(y)$. Also, the conditional mean for a linear budget set $\int y F(d y \mid \rho, R)$ is identified for $(\rho, R) \in \cap_{y} X(y)$.

In many applications the budget set may be nonconvex. It would be useful to know how the CDF depends on the budget set in these cases. We can show that the CDF only depends on $B(y)$ over the the values of $y$ where $B(y)$ is not concave. For simplicity we show this result for the case where $B(y)$ has only one nonconcave segment. Let $[y(B), \bar{y}(B)]$ denote the interval 
where $B(y)$ may not be concave and let $\breve{B}=\left\{y(B), \bar{y}(B),\left.B(z)\right|_{z \in[y(B), \bar{y}(B)]}\right\}$ denote the interval endpoints and the budget frontier over the interval.

Theorem 3: If Assumption 1 and $A 1$ are satisfied then for all $B$ such that $\bar{B}(z)=B(z)$ except possibly for $z \in(y(B), \bar{y}(B))$ and for $y \in[y(B), \bar{y}(B))$ we have $\operatorname{Pr}(y(B, \eta) \leq y)$ depends only on $\breve{B}$.

This result shows that the CDF of taxable income depends on the budget frontier over the entire nonconvex interval, for any point in the interval. Thus, for a piecewise linear budget constraint the CDF would depend on the slope and virtual income of all the segments that affect that nonconvex interval, when $y$ is in that interval.

\section{$5 \quad$ Kinks and Nonparametric Compensated Tax Effects}

Kinks have been used by Saez (2010) and others to provide information about compensated tax effects for small kinks or parametric models. In this section we derive the nonparametric form of a kink probability with general heterogeneity and show how it is related to compensated effects. We also consider in our nonparametric setting how the Slutzky condition is related to a positive kink probability and the density of taxable income being positive.

Consider a kink $\bar{\ell}$ for a piecewise linear budget set where the budget frontier function coincides with that of the convex hull in a neighborhood of the kink and let $\Pi_{\bar{\ell}}$ denote the kink probability. Let $\rho_{-}$and $\rho_{+}$be the slope of the budget set at $\bar{\ell}$ from the left and right respectively. Consider $\rho$ between $\rho_{-}$and $\rho_{+}$and let $R(\rho)=R_{-}+\bar{\ell}\left(\rho_{-}-\rho\right)$ be the virtual income for the linear budget set with slope $\rho$ passing through the kink. Assuming that $y(\rho, R(\rho), \eta)$ is continuously distributed, let

$$
\phi(\rho)=\frac{\partial F(\bar{\ell} \mid \rho, R(\rho))}{\partial y}, \delta(\rho)=\left.E\left[\frac{\partial y(\rho, R, \eta)}{\partial \rho}-\bar{\ell} \frac{\partial y(\rho, R, \eta)}{\partial R} \mid y(\rho, R, \eta)=\bar{\ell}\right]\right|_{R=R(\rho)}
$$

where the expectation is taken over the distribution of $\eta$.

TheOrem 4: If Assumptions 1, A1, and A2 are satisfied then

$$
\Pi_{\bar{\ell}}=\int_{\rho_{+}}^{\rho_{-}} \phi(\rho) \delta(\rho) d \rho \text { and } \phi(\rho) \delta(\rho)=-F_{\rho}(\bar{\ell} \mid \rho, R(\rho))+\bar{\ell} \cdot F_{R}(\bar{\ell} \mid \rho, R(\rho)) .
$$

The $\delta(\rho)$ in Theorem 4 is an average compensated effect of changing $\rho$ for a linear budget set. This compensated effect appears here because virtual income is being adjusted as $\rho$ changes to stay at the kink. The virtual income adjustment needed to remain at the kink corresponds locally to the income adjustment needed to remain on the same indifference curve, as shown 
by Saez (2010). The formula for $\Pi_{\bar{\ell}}$ bears some resemblance to the kink probability formulas in Saez (2010) but differs in important ways. Theorem 4 is global, nonparametric, and takes explicit account of general heterogeneity, unlike Saez (2010) results, that are local or parametric and account for heterogeneity implicitly.

Theorem 4 helps clarify what can be nonparametrically learned from kinks. First, the compensated effects that enter the kink probability are only for individuals that would choose to locate at the kink for a linear budget set with $\rho \in\left[\rho_{+}, \rho_{-}\right]$. Thus, using kinks to provide information about compensated effects is subject to the same issues of external validity as, say, regression discontinuity design (RDD). As RDD only identifies treatment effects for individuals at the jump point so kinks only provide information about compensated effects for individuals that would locate at the kink.

Second, the kink probability depends on both a compensated tax effect $\delta(\rho)$ and on a pure heterogeneity effect $\phi(\rho)$. Intuitively, a kink probability could be large because the compensated tax effect is large or because preferences are distributed in such a way that many like to be at the kink. Information about compensated effects from kinks depends on knowing something about pure heterogeneity effects.

Third, the pure heterogeneity effect, and hence compensated effects, is not identified when $\rho_{-}$and $\rho_{+}$do not vary in the data. One cannot identify the $\operatorname{pdf} \phi(\rho)$ for $\rho \in\left(\rho_{+}, \rho_{-}\right)$because observations will not be available for such $\rho$ values. Because of this it may be impossible to say anything about compensated effects from kinks. An example can be used to illustrate. Suppose that the parameter $\theta$ of interest is a weighted average (over $\rho$ ) of compensated effects $\theta=\int_{\rho_{+}}^{\rho_{-}} \phi(\rho) \delta(\rho) d \rho / \int_{\rho_{+}}^{\rho_{-}} \phi(\rho) d \rho$. Theorem 4 gives

$$
\theta=\frac{\Pi_{\bar{\ell}}}{\int_{\rho_{+}}^{\rho_{-}} \phi(\rho) d \rho} .
$$

Evidently $\theta$ depends on the denominator $\int_{\rho_{+}}^{\rho_{-}} \phi(\rho) d \rho$. If $\rho_{-}$and $\rho_{+}$are fixed then $\phi(\rho)$ is not identified for $\rho \in\left(\rho_{+}, \rho_{-}\right)$so that $\phi(\rho)$ can be anything at all over that interval and the denominator can vary between 0 and $\infty$. In this setting the kink probability provides no information about $\theta$.

If $\phi(\rho)$ is assumed to satisfy certain conditions then a kink probability can provide information about $\theta$ when $\rho_{-}$and $\rho_{+}$are fixed. We can continue to illustrate using the parameter $\theta$. As in Saez $(2010), \phi\left(\rho_{-}\right)$and $\phi\left(\rho_{+}\right)$may be identified from the pdf of taxable income to the left and right of the kink respectively. If $\phi(\rho)$ is assumed to be monotonic for $\rho \in\left(\rho_{+}, \rho_{-}\right)$then we have bounds on $\theta$ of the form

$$
\frac{\Pi_{\bar{\ell}}}{\left(\rho_{-}-\rho_{+}\right) \max \left\{\phi\left(\rho_{-}\right), \phi\left(\rho_{+}\right)\right\}} \leq \theta \leq \frac{\Pi_{\bar{\ell}}}{\left(\rho_{-}-\rho_{+}\right) \min \left\{\phi\left(\rho_{-}\right), \phi\left(\rho_{+}\right)\right\}} .
$$


If $\phi(\rho)$ is assumed to be linear on $\rho \in\left(\rho_{+}, \rho_{-}\right)$then

$$
\theta=\frac{\Pi_{\bar{\ell}}}{\left(\rho_{-}-\rho_{+}\right)\left[\phi\left(\rho_{-}\right)+\phi\left(\rho_{+}\right)\right] / 2} .
$$

Thus we see that assumptions about $\phi(\rho)$ can used to obtain information about $\theta$ from the kink.

In some data the kink may remain fixed while $\rho_{-}$and/or $\rho_{+}$varies. This could occur in a cross section due to variation in local tax rates. In such cases it may be possible to obtain information about $\phi(\rho)$ from the data as $\rho$ varies. This information may then be combined with kink probabilities to obtain information about compensated effects. For brevity we will not consider this kind of information here.

This example of a weighted average compensated effect $\theta$ is meant to highlight the importance of the pure heterogeneity term $\phi(\rho)$ in recovering compensated effects from kink probabilities. Similar issues would arise for measures of compensated effects other than $\theta$. Nonparametrically recovering information about compensated effects from kink probabilities generally requires assuming or knowing something about the pure heterogeneity term.

Theorem 4 can also be used to relate positivity of the kink probability $\Pi_{\bar{\ell}}$ to the Slutzky condition. One could specify a $\operatorname{CDF} F(y \mid \rho, R)$ for taxable income for a linear budget set and derive the probability of a kink from equation (5.5). Then the Slutzky condition is sufficient but not necessary for positivity of $\Pi_{\bar{\ell}}$, because an integral can be positive without the function being integrated being positive. In this sense the kink probability can be positive without all the conditions for utility maximization being satisfied.

A similar thing happens for the pdf of taxable income for a smooth, concave budget frontier. By Theorem 2 the CDF of taxable income for a smooth budget set is $F(y \mid \rho(y), R(y))$. By the chain rule the pdf of taxable income implied by the model will be

$$
\frac{\partial F(y \mid \rho(y), R(y))}{\partial y}=F_{y}(y \mid \rho(y), R(y))+\rho_{y}(y)\left[F_{\rho}(y \mid \rho(y), R(y))-y F_{R}(y \mid \rho(y), R(y))\right],
$$

where $\rho_{y}(y)=\partial \rho(y) / \partial y$. One could specify a $\operatorname{CDF} F(y \mid \rho, R)$ for taxable income for a linear budget set and derive the pdf from equation (5.6). The first term $F_{y}$ is nonnegative because it is a pdf. The $\rho_{y}(y)$ is nonpositive because it is the derivative of the slope of a concave function. Then the Slutzky condition is sufficient for a positive pdf because it means that the second term will be nonnegative, and hence so will the sum. However, the Slutzky condition is not necessary for positivity of the pdf of taxable income because the positivity of the pdf $F_{y}$ can result in positivity of the sum of even when the second term is negative. In this sense the pdf of taxable income for a smooth concave budget frontier can be positive without all the conditions for utility maximization being satisfied. 
This analysis shows that a coherent nonparametric model, one with a positive pdf and kink probabilities, can be constructed without imposing all the conditions of utility maximization. In particular, the distribution of taxable income implied by a particular $F(y \mid \rho, R)$ can be coherent without the Slutzky condition for the CDF being satisfied. This analysis is consistent with most of the comments of Keane (2011) about a previous literature concerning the relationship between positive likelihoods and utility maximization. We do differ in finding that positive kink probabilities are possible without a Slutzky condition, which could be attributed to our nonparametric framework.

\section{The Expected Value of Taxable Income}

The expected value $\mu(B)=\int y(B, \eta) G(d \eta)$ of taxable income for a given budget set is useful for identifying important policy effects. It can be used to predict the effect of tax changes on average taxable income. Furthermore, the presence of an additive mean zero disturbance in taxable income can be allowed for. The presence of such an additive disturbance is one way to account for the common occurrence that individuals do not choose to be at kinks, as noted by Burtless and Hausman (1978).

In this Section we derive $\mu(B)$ for piecewise linear budget sets and show how it can be approximated for estimation purposes. To describe the expected value, recall that $F(y \mid \rho, R)$ is the CDF of taxable income for a linear budget set. Define

$$
\begin{aligned}
\bar{y}(\rho, R) & =\int y F(d y \mid \rho, R), \\
\nu(\rho, R, \ell) & =\int 1(y<\ell)(y-\ell) F(d y \mid \rho, R), \lambda(\rho, R, \ell)=\int 1(y>\ell)(y-\ell) F(d y \mid \rho, R) .
\end{aligned}
$$

These objects are integrals over the $\operatorname{CDF} F(y \mid \rho, R)$ for a linear budget set. The expected value of taxable income given a piecewise linear, convex budget set depends on them in the way shown in the following result:

Theorem 5: If Assumption 1 and $A 1$ are satisfied, $\int|y(\rho, R, \eta)| G(d \eta)<\infty$ for all $\rho, R>0$, $B(y)$ is piecewise linear and concave, and $Y$ is a random variable such that for any $B, E[Y]$ $=\int y(B, \eta) G(d \eta)$, then

$$
\begin{aligned}
\mu(B) & =\bar{y}\left(\rho_{J}, R_{J}\right)+\sum_{j=1}^{J-1}\left[\nu\left(\rho_{j}, R_{j}, \ell_{j}\right)-\nu\left(\rho_{j+1}, R_{j+1}, \ell_{j}\right)\right] \\
& =\bar{y}\left(\rho_{1}, R_{1}\right)+\sum_{j=1}^{J-1}\left[\lambda\left(\rho_{j+1}, R_{j+1}, \ell_{j}\right)-\lambda\left(\rho_{j}, R_{j}, \ell_{j}\right)\right] .
\end{aligned}
$$

The first equality in the conclusion is exactly analogous to the conclusion of Theorem 2.1 of BN. As discussed there, this additive decomposition of the conditional mean makes it feasible 
to nonparametrically estimate the conditional expectation as a function of the budget set. The fact that the conditional expectation only depends on one two-dimensional function $\bar{y}(\rho, R)$ and one three-dimensional function $\nu(\rho, R, \ell)$ (or $\lambda(\rho, R, \ell)$ ) means the curse of dimensionality can be avoided by using a nonparametric estimator that imposes the structure in the formula for $\mu(B)$.

Theorem 5 generalizes Theorem 2.1 of BN by allowing general heterogeneity and zero hours of work, whereas BN assumed scalar $\eta$. Consequently, the empirical conclusions drawn by BN about the average labor supply effect of a large Swedish tax reform are valid under general heterogeneity. To the best of our knowledge that makes the tax policy estimates of BN the first that are valid with general preference heterogeneity.

We can use Theorem 2, which implies that the expectation depends only on the CDF $F(y \mid \rho, R)$ for a linear budget set, to construct a more parsimonious approximation to $\mu(B)$ than BN. The definitions of $\bar{y}(\rho, R)$ and $\nu(\rho, R, \ell)$ (or $\lambda(\rho, R, \ell)$ ) and the conclusion of Theorem 5 give the precise form of the dependence on $F(y \mid \rho, R)$. Replacing $F(y \mid \rho, R)$ by a series approximation in those definitions and plugging the result into the formula in Theorem 5 gives a more parsimonious approximation than $\mathrm{BN}$.

The series approximation we use is a linear in parameters approximation to the conditional CDF of taxable income for a linear budget set. For a positive integer $A$ let $F_{1}(y), \ldots, F_{A}(y)$ be CDF's and $x=(\rho, R)$. Let $r_{1}(x), \ldots, r_{B}(x)$ denote approximating functions, such as splines or polynomials. Let $\beta_{a b},(a=2, \ldots, A ; b=1, \ldots, B)$ be coefficients of a series approximation to be specified below and $w_{a}(x, \beta)=\sum_{b=1}^{B} \beta_{a b} r_{b}(x)$. We consider an approximation to the conditional $\mathrm{CDF}$ of the form

$$
\begin{aligned}
F(y \mid x) & \approx F_{1}(y)+\sum_{a=2}^{A} w_{a}(x, \beta)\left[F_{a}(y)-F_{1}(y)\right] \\
& =\sum_{a=1}^{A} w_{a}(x, \beta) F_{a}(y), w_{1}(x, \beta)=1-\sum_{a=2}^{A} w_{a}(x, \beta) .
\end{aligned}
$$

This could be thought of as a mixture approximation to the conditional CDF with weights $w_{a}(x, \beta), a=1, \ldots, A$. We have normalized the weights to sum to one by choosing $w_{1}(x, \beta)$ as above. Because of this normalization the conditional CDF approximation will go to 1 as $y$ grows for all $x$. We do not impose that the weights $w_{a}(x, \beta)$ are nonnegative. We are primarily interested in approximating the expected value and so are not concerned that the underlying approximation to the conditional CDF be everywhere increasing.

We obtain an approximation to the conditional mean by plugging the CDF approximation into the respective formulas for $\bar{y}(\rho, R)$ and $\nu(\rho, R, \ell)$ and then into the formula for the mean 
in Theorem 5. Let

$$
\bar{y}_{a}=\int y F_{a}(d y), \nu_{a}(\ell)=\int 1(y<\ell)(y-\ell) F_{a}(d y),(a=1, \ldots, A) .
$$

Substituting the CDF approximation in the expression for the conditional mean from Theorem 5 gives

$$
\begin{aligned}
\mu(B) & \approx \bar{y}_{1}+\sum_{a=2}^{A} w_{a}\left(x_{J}, \beta\right)\left(\bar{y}_{a}-\bar{y}_{1}\right)+\sum_{a=2}^{A} \sum_{j=1}^{J-1}\left[w_{a}\left(x_{j}, \beta\right)-w_{a}\left(x_{j+1}, \beta\right)\right]\left[\nu_{a}\left(\ell_{j}\right)-\nu_{1}\left(\ell_{j}\right)\right] \\
& =\bar{y}_{1}+\sum_{a=2}^{A} \sum_{b=1}^{B} \beta_{a b}\left\{r_{b}\left(x_{J}\right)\left(\bar{y}_{a}-\bar{y}_{1}\right)+\sum_{j=1}^{J-1}\left[r_{b}\left(x_{j}\right)-r_{b}\left(x_{j+1}\right)\right]\left[\nu_{a}\left(\ell_{j}\right)-\nu_{1}\left(\ell_{j}\right)\right]\right\} .
\end{aligned}
$$

This is a series approximation, where the regressor corresponding to $\beta_{a b}$ is a linear combination of the approximating function evaluated on the last segment and differences of approximating functions between segments. A series estimator can be obtained by running least squares of $y_{i}$ on these regressors.

A series estimator based on this approximation imposes the restrictions that the same CDF for a linear budget set appears in both $\bar{y}\left(\rho_{J}, R_{J}\right)$ and in $\nu(\rho, R, \ell)$. This approximation is more parsimonious than BN (based on Theorem 5) because it does not use a separate approximation to $\bar{y}(\rho, R)$. It makes use of Theorem 2 , being based on an approximation to the CDF of taxable income for a linear budget set, which is the underlying nonparametric object determining the distribution of taxable income.

By Theorem 1 the one additional restriction imposed by utility maximization is that the CDF for a linear budget set satisfies the Slutzky condition. It is straightforward to impose this restriction on a grid of values for $x$ and $y$, say $x_{1}, \ldots, x_{C}$, and $y_{1}, \ldots, y_{D}$. The CDF of taxable income for a linear budget set with slope and intercept $x$ that corresponds to this approximation is $F_{1}(y)+\sum_{a=2}^{A} w_{a}(x, \beta)\left[F_{a}(y)-F_{1}(y)\right]$. The Slutzky condition for the CDF approximation at the values of $x$ and $y$ is then

$$
\sum_{a=2}^{A}\left[\frac{\partial w_{a}\left(x_{c}, \beta\right)}{\partial \rho}-y_{d} \frac{\partial w_{a}\left(x_{c}, \beta\right)}{\partial R}\right]\left[F_{a}\left(y_{d}\right)-F_{1}\left(y_{d}\right)\right] \leq 0,(c=1, \ldots, C ; d=1, \ldots, D) .
$$

These are a set of linear in parameters, inequality restrictions on the coefficients $\beta$ of the weights $w_{a}(x, \beta)$. A series approximation as above with coefficients satisfying these Slutzky inequalities is an approximation to the expected value that approximately satisfies all the restrictions of utility maximization. Because the only restriction imposed by utility maximization are the Slutzky condition being satisfied for $F(y \mid x)$ we know that approximately imposing all those conditions approximately imposes all the conditions of utility maximization. 
To show that this approximation works we give a rate result for specific types of CDF's $F_{a}(y)$ and functions $r_{b}(x)$. We view this result as a theoretical justification of the approach though it may not be the best for applications. In our application we use different CDF's that are more closely linked to the data we have. The rate result is based on choosing $F_{a}(y)$ to be integrals of b-splines that are positive and normalized to integrate to one and on $r_{b}(x)$ also being splines. We also require that $y$ and $x$ are contained in bounded sets $\mathcal{Y}$ and $\mathcal{X}$ and that the conditional pdf of taxable income for a linear budget set $f(y \mid x)$ be smooth.

Theorem 6: If $\mathcal{Y}$ and $\mathcal{X}$ are compact, $f(y \mid x)$ is zero outside $\mathcal{Y} \times \mathcal{X}$ and is continuously differentiable to order $s$ on $\mathcal{Y} \times \mathcal{X}$, and $d F_{a}(y) / d y,(a=1, \ldots, A)$ and $r_{b}(x),(b=1, \ldots, B)$ consist of tensor product b-splines of order $s$ on $\mathcal{Y} \times \mathcal{X}$ then there exist a constant $C$ and $\beta_{a b}$ such that for all piecewise linear, concave budget frontiers with $x_{j} \in \mathcal{X},(j=1, \ldots, J)$,

$$
\begin{aligned}
& \left|\mu(B)-\bar{y}_{1}-\sum_{a=2}^{A} \sum_{b=1}^{B} \beta_{a b}\left\{r_{b}\left(x_{J}\right)\left(\bar{y}_{a}-\bar{y}_{1}\right)+\sum_{j=1}^{J-1}\left[r_{b}\left(x_{j}\right)-r_{b}\left(x_{j+1}\right)\right]\left[\nu_{a}\left(\ell_{j}\right)-\nu_{1}\left(\ell_{j}\right)\right]\right\}\right| \\
\leq & C A^{1-s} B^{(1-s) / 2} .
\end{aligned}
$$

This result shows that the series approximation we have proposed does indeed approximate the expected value of taxable income for concave piecewise linear budget frontiers. The approximation rate is uniform in the number $J$ of budget segments. The rate of approximation corresponds to a multivariate B-spline approximation to a function and its derivative, where approximating the derivative is useful for making the rate uniform in the number of budget segments.

We can also make allowance for nonconcave budget frontiers. For example, suppose that the budget frontier is nonconcave over only two segments. From Theorem 3 we see that the distribution over those segments will depend only on the slope and intercept of those two segments. Because the expected value is a sum of integrals over different segments it would take the form

$$
\mu(B)=\bar{y}\left(\rho_{J}, R_{J}\right)+\sum_{j=1}^{J-1}\left[\nu\left(\rho_{j}, R_{j}, \ell_{j}\right)-\nu\left(\rho_{j+1}, R_{j+1}, \ell_{j}\right)\right]+\varsigma\left(R_{\tilde{j}-1}, \rho_{\tilde{j}-1}, R_{\tilde{j}}, \rho_{\tilde{j}}\right),
$$

where $\tilde{j}$ and $\tilde{j}-1$ index the segments where the nonconcavities occur. The $\varsigma$ term represents the deviation of the mean from what it would be if the budget frontier were concave. It can be accounted for in the approximation by separately including series terms that depend just on $R_{\tilde{j}-1}, \rho_{\tilde{j}-1}, R_{\tilde{j}}$, and $\rho_{j}$. If the nonconcavities are small or few people have taxable income where they occur then $\varsigma$ would be small and including terms to account for $\varsigma$ will lead to little improvement. The integration across individuals to obtain the expected value reduces the importance of nonconcavities. 


\section{$7 \quad$ Policy Effects and Productivity Growth}

It is common practice to measure behavioral effects in terms of elasticities. We are used to linear budget constraints and elasticities with respect to the net of tax rate and non-labor income of a linear budget constraint. One problem with nonlinear budget constraints is that this elasticity may not be identified. The elasticity for a linear budget constraint would often be thought of as corresponding to $\bar{y}(\rho, R)$. From the discussion following Theorem 2 we see that this function is only identified where for every $y$ the value of $\rho$ is the (right) slope of a budget frontier at $y$ for some budget set available in the data. The set of such net of tax rates could well be very small, even empty. Therefore we must look for other kinds of elasticities to hope for identification. Furthermore, since everyone generally faces a nonlinear budget set, and policy changes are not likely to eliminate this nonlinearity, it makes sense to focus on effects of changes in a nonlinear budget set.

For motivation we first consider effects for the average taxable income $\bar{y}(\rho, R)$ for a linear budget set. As usual, the average net-of-tax effect will be $d \bar{y}(\rho, R) / d \rho$ and the average effect of nonlabor income will be $d \bar{y}(\rho, R) / d R$. Next consider the case where the expected taxable income is a function of a piecewise-linear budget constraint, say $\mu(B)=g\left(\rho_{1}, \ldots, \rho_{J}, R_{1}, \ldots, R_{J}\right)$ for a function $g$. Assume that the budget constraint is continuous so that the kink points will be well defined by the net-of-tax rates and virtual incomes and are given by $\ell_{j}=\left(R_{j}-R_{j+1}\right) /\left(\rho_{j+1}-\rho_{j}\right)$. Let $G(a, b)=g\left(\rho_{1}+a, \ldots, \rho_{J}+a, R_{1}+b, \ldots, R_{J}+b\right)$. The parameter $a$ tilts the budget constraint, and the parameter $b$ shifts the budget constraint vertically, both while holding fixed the kink points. For policy purposes $a$ is like a change in a local proportional tax rate, and $b$ is like a change in unearned income. Identification of effects for changes in $a$ and $b$ only requires variation in the overall slopes and intercepts of the budget constraint across individuals and time periods. This is a common source of variation in nonlinear budget sets due to variations in local tax rates and in nonlabor income, so effects of such a change should be identified.

Consider the derivative of $G(a, b)$ with respect to $a$ evaluated at $a=b=0$, given by $\partial G / \partial a=\sum_{j=1}^{J} \partial g / \partial \rho_{j}$. This is the effect on the expectation of tilting the budget constraint. To obtain an elasticity we multiply this derivative by a constant $\tilde{\rho}$ that represents the vector of net-of-tax rates by a single number and then divide by $\mu(B)$. The construction of $\tilde{\rho}$ can be done in many different ways. We use the sample averages of the net-of-tax rates and virtual incomes for the segments where individuals are actually located. Our elasticity $(\partial G / \partial a)(\tilde{\rho} / E[Y])$ is an aggregate elasticity which is the policy relevant measure as argued in Saez et. al. (2012). We also consider the effect of unearned income $R$ given by $\partial G / \partial b=\sum_{j=1}^{J} \partial g / \partial R_{j}$. Due to ambiguity on how to normalize this we do not report an elasticity. Instead we will simply 
report estimates of the unearned income effect $\partial G / \partial b$.

In the long run, exogenous wage growth is a major determinant of individuals' real incomes. Such growth may be caused by factors such as technological development, physical capital, and human capital. It is important to account for such growth when identifying the effects of taxes on taxable income using variation over time as we do in the application below. We do so by assuming that productivity growth is the same in percentage terms for all individuals. We assume the wage rate in period $t$ is given by $w=g(e, \eta) \phi(t)$ with $\phi(0)=1$. The function $\phi(t)$ is a function that captures exogenous productivity growth, i.e., percentage changes in an individual's wage rate that do not depend on the individual's behavior.

With productivity growth and heterogeneity the individual's optimization problem is: ${ }^{3}$

$$
\operatorname{Max} u(c, e, h, \eta) \quad \text { s.t. } \quad c=g(e, \eta) \phi(t) h \rho+R
$$

This problem can be solved similarly to previous ones, by letting $y=w h$, inverting the wage function, and choosing hours of work to maximize $u\left(c, g^{-1}(y /(h \phi(t)), \eta), h, \eta\right)$ over $h$. Concentrating out hours of work gives the concentrated utility function $U(c, y / \phi(t), \eta)$. In a second step the individual solves $\operatorname{Max} U(c, y / \phi(t), \eta)$ s.t. $c=y \rho+R$.

A feature of this problem is that the concentrated utility shifts over time. Our approach to repeated cross section data depends on using a preference specification invariant to individuals and time. A simple way to do that is to focus on taxable income net of productivity growth, given by $\tilde{y}=y / \phi(t)$. Then the reduced-form maximization problem becomes $\operatorname{Max} U(c, \tilde{y}, \eta)$ s.t. $c=\tilde{y} \tilde{\rho}+R$ for $\tilde{\rho}=\phi(t) \theta$. Here the productivity growth appears in the budget set, multiplying the net of tax rate. From the tax authorities' point of view the taxable income is $y=\phi(t) \tilde{y}$. However, to keep things stationary over time we study the behavior of $\tilde{y}$.

Although the function $U(c, \tilde{y}, \eta)$ does not shift over time, it depends on a base year and a normalization of $\phi(0)$ to one. If we use another base year we would have another concentrated utility function $U(c, y, \eta)$.

This way to account for productivity growth is similar to that used in log-linear models. Suppose that $y=[\phi(t) \rho]^{\beta} \eta$, where $\beta$ is the net-of-tax elasticity of interest and that there are no income effects. Taking logarithms gives $\ln y=\beta \ln \phi(t)+\beta \ln \rho+\ln \eta$. Here $\phi(t)$ enters as a time effect and $\beta$ can be identified in a regression involving the logarithm of the uncorrected variables $y$ and $\rho$. This is, more or less, how productivity growth has been accounted for in previous models. Including time effects in log-linear models corresponds to the productivity

\footnotetext{
${ }^{3}$ Note that we are still considering an atemporal model of individual behavior. An individual considers a sequence of one-period optimization problems. The purpose of the extension here is to show how to account for exogenous productivity growth.
} 
growth specification we adopt here.

To implement the corrections on the net-of-tax rates and the dependent variable we need to know the wage/productivity growth. Unfortunately there are few good measures of the exogenous wage/productivity growth. The productivity measures available in the literature have in general not separated out the change in wages that is due to behavioral effects of tax changes. We will therefore use our data to estimate exogenous wage growth. To not use up too much identifying information when doing this we constrain the annual productivity growth to be the same every year, where $\phi(t)=e^{g t}$ for some constant $g$. This may well be misspecified. However, to do a more refined correction of the budget constraints would use up much of the information in the data. In particular we would lose much of the identifying power of changes in the overall tax rate across years. We do not think there are wide swings in the productivity growth rate from year to year so that the misspecification would not be very large for any individual year's budget constraints.

In the long run changes in tax rates can be swamped by productivity growth. For example, over say a twenty-year period, if the annual productivity growth is $0.02, \phi(20) / \phi(0)$ will be 1.5 , corresponding to an increase in the net-of-tax rate of a factor of 1.5. In the short run, changes in tax rates can swamp short-run changes in $\phi(t)$. For example, a change in the tax rate from, say, 0.6 to 0.4 raises $\rho$ by a factor of 1.5 .

In a linear budget set, productivity growth and tax-rate changes have the same kind of effect on net-of-tax rates. It can therefore be difficult to nonparametrically separate the two kinds of effects. In a nonlinear budget set the situation is different. Consider an example with two budget segments. The budget constraint can then be written as $c=\tilde{y} \phi(t) \rho_{1}+R_{1}$ for $\tilde{y}<\phi(t)^{-1} \ell_{1}$ and $c=\tilde{y} \phi(t) \rho_{2}+R_{2}$ for $\tilde{y}>\phi(t)^{-1} \ell_{1}$. In this specification productivity changes shift both slopes and kinks, a different effect than just a change in slopes. These effects are also present for budget sets with many segments. Thus, productivity changes have different effects on the budget sets than just changing slopes, so it may be possible to separate out the effect of productivity growth and tax rate changes in our estimates.

It would be interesting to allow for the productivity growth rate to vary with individuals, i.e. to allow for heterogeneity in productivity growth. We do this in the empirical application by allowing productivity growth to vary between more and less educated individuals. We find that allowing this observed heterogeneity does not impact our tax and income effect estimates. We could also allow for unobserved heterogeneity by specifying that $c=g(e, \eta) \phi(t, \eta) h \rho+R$. Unfortunately this does not lead to a stable utility function over time and so would not allow us to use time variation in taxes to identify tax effects. This variation appears to be important for the ability to estimate tax effects in our data so we do not pursue models with unobserved 
heterogeneity in productivity growth.

\section{Empirical Application}

The previous results are based on the expected value and distribution of taxable income for a given budget frontier. These objects are identified when the budget sets in the data are independent of preferences. We can also allow for an additive disturbance with mean zero. That is, suppose that that the data are consist of observations on taxable inocme and budget frontiers for individuals $\left(Y_{i}, B_{i}\right),(i=1, \ldots, n)$ with $Y_{i}=y\left(B_{i}, \eta_{i}\right)+\epsilon_{i}, B_{i}$ and $\eta_{i}$ are statistically independent, and $E\left[\epsilon_{i} \mid B_{i}\right]=0$. In that case

$$
E\left[Y_{i} \mid B_{i}=B\right]=\mu(B) .
$$

Here the expected value of taxable income for a given frontier is the conditional expectation of taxable income in the data. The expected value can then be estimated by the nonparametric series estimator described earlier. Specifically, for $\bar{y}_{a}, \nu_{a}(\ell)$, and $r_{b}(x)$ as previously defined, $Y_{i}-\bar{y}_{1}$ could be regressed on

$$
r_{b}\left(x_{i, J_{i}}\right)\left(\bar{y}_{a}-\bar{y}_{1}\right)+\sum_{j=1}^{J_{i}-1}\left[r_{b}\left(x_{i j}\right)-r_{b}\left(x_{i, j+1}\right)\right]\left[\nu_{a}\left(\ell_{i j}\right)-\nu_{1}\left(\ell_{i j}\right)\right], a=2, \ldots, A ; b=1, \ldots, B,
$$

where $J_{i}$ is the number of budget segments for the $i^{t h}$ observation, $x_{i j}=\left(\rho_{i j}, R_{i j}\right)$ is the slope and intercept of the $j^{\text {th }}$ segment, and $\ell_{i j}$ is the location of the $j^{\text {th }}$ knot. For the coefficients $\hat{\beta}_{a b}$ obtained from this regression, the estimator of the expected value of taxable income for any concave piecewise linear budget frontier is then

$$
\hat{\mu}(B)=\bar{y}_{1}+\sum_{a=2}^{A} \sum_{b=1}^{B} \hat{\beta}_{a b}\left\{r_{b}\left(x_{J}\right)\left(\bar{y}_{a}-\bar{y}_{1}\right)+\sum_{j=1}^{J-1}\left[r_{b}\left(x_{j}\right)-r_{b}\left(x_{j+1}\right)\right]\left[\nu_{a}\left(\ell_{j}\right)-\nu_{1}\left(\ell_{j}\right)\right]\right\} .
$$

Independence of $\eta_{i}$ and $B_{i}$ can be relaxed to allow for covariates and/or control functions. For covariates $w$ we consider an index specification where there is a vector of functions $v(w, \delta)$ such that $\eta_{i}$ and $\left(B_{i}, v\left(w_{i}, \delta_{0}\right)\right)$ are independent for some parameter value $\delta_{0}$. These covariates might include demographic variables that represent observed components of the utility. For example, one could use a single, linear index $v(w, \delta)=w_{1}+w_{2}^{T} \delta$, with the usual scale and location normalization imposed. Covariates can be allowed by letting the functions $r_{b}(x, v)$ depend on the index $v$ as well as the slope and intercept $x$. This specification corresponds to preferences that are allowed to depend on $v\left(w, \delta_{0}\right)$. The parameters $\beta_{a b}$ and $\delta$ could then be 
estimated by nonlinear least squares, as the minimizers of

$$
\begin{aligned}
& \sum_{i=1}^{n}\left\{Y_{i}-\bar{y}_{1}-\sum_{a=2}^{A} \sum_{b=1}^{B} \beta_{a b}\left\{r_{b}\left(x_{i, J_{i}}, v\left(w_{i}, \delta\right)\right)\left(\bar{y}_{a}-\bar{y}_{1}\right)\right.\right. \\
& \left.\left.+\sum_{j=1}^{J_{i}-1}\left[r_{b}\left(x_{i j}, v\left(w_{i}, \delta\right)\right)-r_{b}\left(x_{i, j+1}, v\left(w_{i}, \delta\right)\right)\right]\left[\nu_{a}\left(\ell_{j}\right)-\nu_{1}\left(\ell_{j}\right)\right]\right\}\right\}^{2} .
\end{aligned}
$$

An estimable control variable can be used to account for endogeneity. Such a control variable would be $\xi_{i}$ such that $B_{i}$ and $\eta_{i}$ are independent conditional on $\xi_{i}$ and the conditional support of $\xi_{i}$ given $B_{i}$ equals the marginal support of $\xi_{i}$. In that case it follows as in Blundell and Powell (2006) that

$$
\int E\left[Y_{i} \mid B_{i}=B, \xi_{i}=\xi\right] F_{\xi}(d \xi)=\mu(B)
$$

where $F_{\xi}(\xi)$ is the CDF of $\xi_{i}$. This integral can be estimated by letting the functions $r_{b}(x, \xi)$ depend on $\xi$ and then including $\hat{\xi}_{i}$ to form the regressors

$$
r_{b}\left(x_{i, J_{i}}, \hat{\xi}_{i}\right)\left(\bar{y}_{a}-\bar{y}_{1}\right)+\sum_{j=1}^{J_{i}-1}\left[r_{b}\left(x_{i j}, \hat{\xi}_{i}\right)-r_{b}\left(x_{i, j+1}, \hat{\xi}_{i}\right)\right]\left[\nu_{a}\left(\ell_{i j}\right)-\nu_{1}\left(\ell_{i j}\right)\right], a=2, \ldots, A ; b=1, \ldots, B .
$$

The expected value of taxable income could then be estimated by averaging over the observations $\hat{\xi}_{i}$, with

$$
\begin{aligned}
\hat{\mu}(B) & =\bar{y}_{1}+\sum_{a=2}^{A} \sum_{b=1}^{B} \hat{\beta}_{a b}\left\{\bar{r}_{b}\left(x_{J}\right)\left(\bar{y}_{a}-\bar{y}_{1}\right)+\sum_{j=1}^{J-1}\left[\bar{r}_{b}\left(x_{j}\right)-\bar{r}_{b}\left(x_{j+1}\right)\right]\left[\nu_{a}\left(\ell_{j}\right)-\nu_{1}\left(\ell_{j}\right)\right]\right\}, \\
\bar{r}_{b}(x) & =\sum_{i=1}^{n} r_{b}\left(x, \hat{\xi}_{i}\right) / n .
\end{aligned}
$$

Although conditions for existence of a control variable are quite strong (see Blundell and Matzkin, 2014), this approach does provide a way to allow for some forms of endogeneity.

In the application we use $F_{a}(y)$ that are constructed from the data in a way described in the next Section. We use power series terms for $r_{b}(x, v, \xi)$, with

$$
r_{b}(x, v, \xi)=\rho^{m_{1}(b)} R^{m_{2}(b)} v^{m_{3}(b)} \xi^{m_{4}(b)},
$$

where $m_{k}(b)$ are nonnegative integers.

An important problem for practice is the selection of which approximating functions to include in estimation. For this purpose we use a hybrid of standard Lasso and cross-validation approaches to model selection. To describe the method let $i$ index the observations and $p_{i}^{K}=$ $\left(p_{i 1}^{K}, \ldots, p_{i K}^{K}\right)^{\prime}$ be the vector of approximating functions, say from equation (8.10) when there 
are no covariates or control functions. Here $K$ denotes the number of approximating functions, which will be $(A-1) B$ in the case of equation (8.10). We adopt an approach similar to Belloni and Chernozhukov (2013) in using Lasso as a model selection method. The goal is to select a subvector of $p_{i}^{K}$ to use in the least squares estimation described above. Note that this process begins with a choice of $K$, which is generally reasonably large. The regressors need to be normalized so that they all have sample second moment equal to one. This can be done by diving each observation $p_{i k}^{K}$ on the $k^{t h}$ regressor by $\left\{\sum_{i=1}^{n}\left(p_{i k}^{K}\right)^{2} / n\right\}^{1 / 2}$. Here is a multi-step description of the model selection method we use:

a) Get a first estimate of $\hat{\mu}(B)$ by using all $K$ terms, where $K$ is chosen by cross-validation or some other method.

b) Calculate the sample residuals $\hat{\varepsilon}_{i}=Y_{i}-\hat{\mu}\left(B_{i}\right)$.

c) Draw a sample $\left(\hat{\varepsilon}_{1}^{b}, \ldots, \hat{\varepsilon}_{n}^{b}\right)$ of size $n$ from the empirical distribution of the residuals.

d) Calculate $\hat{S}_{b}=2 \max _{k \leq K}\left|\sum_{i=1}^{n} p_{i k}^{K} \hat{\varepsilon}_{i}^{b} / n\right|$.

e) Repeat c) and d) $B$ times to obtain $\hat{S}_{1}, \ldots, \hat{S}_{B}$. We could set $B$ to be a few hundred, say 500 , to help estimate the .95 quantile of the distribution of $\hat{S}$.

f) Choose $\hat{S}$ to be the $B * .95$ order statistic of $\hat{S}_{1}, \ldots, \hat{S}_{B}$. This is an estimate of the .95 quantile of the distribution of $\hat{S}$.

g) Let $\hat{\lambda}=c n \hat{S}$ where $c>1$. We use $c=2$ and try different values of $c$, mostly smaller.

h) Minimize

$$
\frac{\sum_{i=1}^{n}\left(Y_{i}-p_{i k}^{K \prime} \beta\right)^{2}}{n}+\frac{\hat{\lambda}}{n} \sum_{j=1}^{J}\left|\beta_{j}\right| .
$$

using the Matlab program for lasso.

i) Do OLS using only those elements of $p_{i k}^{K}$ which had nonzero coefficients in the previous step.

j) Try other values of $\lambda$ as suggested in g) above corresponding to $c$ closer to 1 and slightly bigger than 2 .

k) For each choice of $\lambda$ compute the cross-validation criteria using the OLS estimates from i) for each model, to provide some goodness of fit comparisons.

Once we have estimated coefficients $\hat{\beta}_{a b}$ in hand by following the above procedure, we can construct taxable income elasticities and income effects in the way described previously. It is straightforward to construct the nonlinear budget set effects described earlier from the estimated parameters for a given budget set, control function, and residual function. We average over the sample distribution of budget sets, single-index and control functions to obtain an average effect. We also use an analogous procedure to estimate the effect of some tax reforms on the expected value of taxable income. We estimate standard errors using the delta method. 


\section{Application to Sweden}

\subsection{Data}

We use data from HEK (Hushållens Ekonomi) provided by Statistics Sweden, which is a combined register and survey data set. The data set contains repeated cross sections of approximately 17,000 randomly-sampled individuals from the population and members of their households each year. The response rate is approximately seventy percent. The register component contains income, tax, and demographic data used by the authorities for taxation purposes. The survey component primarily contains housing variables required to construct several housingrelated budget set variables such as the housing allowance, which are important components of the budget sets.

We use data covering a period of sixteen years, from 1993 to 2008. In the estimation, we limit the sample to married or cohabiting men between 21 and sixty years of age. This is the economically most significant group with respect to labor income. We exclude those receiving medical-leave benefits, parental benefits, income from self-employment, or student financial aid above half of the average monthly gross labor income, which was 17,607 SEK in 2008. We use this limit instead of zero, as that would result in a large loss of observations. Out of 102,630 married or cohabiting men between 21 and sixty years of age, 81,718 observations remain after this sample restriction.

Our labor income definition primarily includes third-party reported earned income and income from self-employment. It excludes, however, medical-leave benefits and parental benefits, unlike previous studies using Swedish data. Note that those individuals with large amounts of income from these sources are excluded from the sample.

To construct the individual budget sets, we use a micro simulation model, FASIT, developed by Statistics Sweden, which in principle captures all of the features of the Swedish tax and transfer system relevant for individuals. FASIT is used by, e.g., the Swedish Ministry of Finance, to simulate the mechanical effects of various tax policies including potential future policies. Single cross sections of this model have been previously employed by Flood et al. (2007), Aaberge and Flood (2008), and Ericson et al. (2009). We construct the budget sets by iteratively letting FASIT calculate net family incomes by varying individuals' gross labor incomes. When doing this, we set medical-leave compensation to zero as this is a component that is difficult to predict for the individuals in the beginning of the year when planning how much to work during the year.

We set non-labor income as the net income the family would receive if the husband had no labor income. This component includes the spouse's net labor income, family's net capital 
income, and various welfare benefits the family would receive if the husband had no labor income. For capital incomes, we set capital gains and losses to zero for the same reason as we set sick leave benefits to zero. Additionally, we include the implicit income from residence-owned housing in nonlabor income.

Nonlabor income may be endogenous. We instrument nonlabor income using transfers received at zero labor income. This includes, e.g., housing and child allowances and social assistance. Like the tax system, the transfer system is beyond the control of individuals. The transfers that would be received at zero labor income vary between individuals depending on demographics. Because we control for such factors, most of the variation arises due to changes in the transfer system between years and how these changes affect different individuals differently. The control variable we use is the residual from a linear regression of nonlabor income on the instrument and the demographic variables described below.

We also adjust the budget sets for indirect taxation. Payroll taxes are generated by FASIT, while we make a simple rudimentary correction for consumption taxes using the quotient of aggregate value-added-tax revenues divided by aggregate private consumption for each year separately. These additional corrections are similar to those in Blomquist and Newey (2002).

The data set contains many demographic background variables. We control for age (eight groups), educational level (seven groups), socioeconomic occupational groups (eight groups), spousal income (twenty groups), county of residence (22 groups), whether the individual has children below age six, and whether he is born abroad. These variables are all included as covariates in the index function described above.

In Table B1 in Appendix B, we report sample statistics. We report the mean values of gross labor income, some variables characterizing the budget sets, some demographic variables, and the instrument for nonlabor income. We report statistics for the entire sample, as well as for the years 1993, 1998, 2003, and 2008 separately, to illustrate the development over time.

\subsection{Results}

We do nonparametric estimation using a series approximation with selection among series terms up to a fourth order using the procedures described in Section 8. For the approximation we use three CDF's $F_{a}(y),(a=1,2,3)$, corresponding to the marginal empirical distribution of taxable income for the whole sample, for the smallest $1 / 5$ of the sample, and for the largest $1 / 5$ of the sample. For constructing $\mu_{a}(\ell)$ we use the sample CDF for a subsample of 5,000 observations evenly spaced by gross labor income rank.

We report the net-of-tax elasticity described in Section 7. The marginal effect is evaluated at each of the sample individual budget sets and we report the sample average marginal effects 
scaled by the sample mean income and marginal net-of-tax rate. We report the marginal effect for the income effect, i.e., the change in gross labor income as net nonlabor income increases by one SEK. We do this because the income elasticity is highly dependent on the scaling parameters. The natural scaling factor, the average marginal virtual income is extremely high in our case compared to other studies that report income elasticities because the marginal tax rate is low and nonlabor income is high. Nonlabor income is high because we include the spouse's net income and implicit income from residence-owned housing. The marginal income effect is therefore more informative.

We implicitly estimate the productivity growth rate by estimating specifications assuming different productivity growth rates and by selecting the specification that maximizes the crossvalidation value. We vary the productivity growth rate between $0 \%$ and $1.4 \%$ in steps of $0.1 \%$ when doing this. In Table 1, we report the estimated cross-validation values at different growth rates and specifications with different Lasso lambdas. We normalize so that larger cross-validation values correspond to better fits.

Table 1: Cross-validation Values

\begin{tabular}{c|cccccc}
\hline Growth/Lambda & 2 & 1.5 & 1 & 0.5 & 0.2 & 0.1 \\
\hline $0 \%$ & 0.1003 & 0.0891 & 0.0945 & 0.1026 & -0.6284 & -0.2095 \\
$.1 \%$ & 0.1001 & -1.3758 & -1.3758 & 0.0915 & -0.1517 & -3.95 \\
$.2 \%$ & 0.0987 & 0.1039 & 0.1039 & 0.0106 & -2.4467 & -10.82 \\
$.3 \%$ & 0.0968 & 0.1067 & 0.1067 & 0.1112 & -0.1563 & -0.0227 \\
$.4 \%$ & 0.0945 & 0.0997 & 0.0997 & -0.583 & -10.471 & -1.8175 \\
$.5 \%$ & 0.0919 & 0.0933 & 0.0933 & 0.1036 & -1.3474 & -9.5351 \\
$.6 \%$ & 0.0889 & 0.0942 & -1.9278 & -0.0397 & 0.0728 & -1.0969 \\
$.7 \%$ & 0.0858 & 0.0678 & 0.0824 & 0.1004 & 0.0773 & -0.4132 \\
$.8 \%$ & 0.0824 & 0.0878 & 0.0878 & 0.0702 & 0.1095 & 0.0028 \\
$.9 \%$ & 0.0756 & -0.0432 & -0.0432 & 0.0916 & -0.7532 & -0.4593 \\
$1.0 \%$ & 0.0755 & 0.0807 & 0.0807 & 0.0699 & 0.107 & -0.9698 \\
$1.1 \%$ & 0.072 & 0.0773 & 0.0771 & 0.0809 & -2.6009 & -0.0756 \\
$1.2 \%$ & 0.0401 & 0.083 & 0.083 & 0.073 & -0.8163 & -0.4652 \\
$1.3 \%$ & 0.0649 & 0.07 & 0.0683 & 0.0792 & -0.0764 & -22.41 \\
$1.4 \%$ & 0.0518 & 0.0671 & 0.0409 & 0.067 & -0.9842 & -0.4108 \\
\hline
\end{tabular}

We observe that the cross-validation criterion is maximized at the productivity growth rate $.3 \%$ (at Lasso lambda .5). Gross labor income increased by a factor of 1.23 during the sample period implying an average geometric annual growth rate of $1.4 \%$. The way we have estimated productivity growth here means that we would attribute most of the increase in gross labor income to average responses to changes in the tax structure.

In Table 2, we report the net-of-tax elasticity point estimates in the different specifications. The elasticities are evaluated at the sample mean income of 420,329 SEK and marginal net-oftax rate of .32. Standard errors constructed using the delta method are either .08 or .09 in all 
specifications. We observe that the estimated elasticity decreases as we increase the productivity growth rate. This reflects that, overall, taxes have decreased over the sample period at the same time as gross labor incomes have increased. Using a specification that assumes a too low (high) productivity growth rate (such as assuming no growth) would therefore results in a positive (negative) bias in the estimated elasticities. Accounting for productivity growth appropriately is therefore important. At the specification with largest cross validation value the net-oftax elasticity is .21 and statistically significant, with a $.3 \%$ growth rate. This is roughly the same elasticity found by Blomquist and Selin (2010). The highest cross validation values give estimated net-of-tax elasticities of 0.38 for a growth rate of $0.2 \%$ and 0.07 for a slightly higher growth rate of $.4 \%$.

Table 2: Estimated Net of Tax Elasticities.

\begin{tabular}{c|cccccc}
\hline Growth/Lambda & 2 & 1.5 & 1 & 0.5 & 0.2 & 0.1 \\
\hline $0 \%$ & 0.76 & 0.71 & 0.71 & 0.68 & 0.72 & 0.79 \\
$.1 \%$ & 0.62 & 0.55 & 0.55 & 0.55 & 0.56 & 0.62 \\
$.2 \%$ & 0.38 & 0.38 & 0.38 & 0.37 & 0.36 & 0.46 \\
$.3 \%$ & 0.22 & 0.52 & 0.52 & 0.21 & 0.27 & 0.25 \\
$.4 \%$ & 0.07 & 0.07 & 0.07 & 0.05 & 0.68 & 0.05 \\
$.5 \%$ & -0.05 & -0.05 & -0.05 & -0.07 & -0.07 & 0.6 \\
$.6 \%$ & 0.05 & -0.15 & -0.17 & -0.07 & -0.14 & -0.16 \\
$.7 \%$ & -0.21 & -0.22 & -0.2 & -0.21 & -0.21 & -0.24 \\
$.8 \%$ & -0.14 & -0.25 & -0.25 & -0.24 & -0.23 & -0.23 \\
$.9 \%$ & -0.24 & -0.24 & -0.24 & -0.25 & -0.22 & -0.25 \\
$1.0 \%$ & -0.29 & -0.29 & -0.29 & -0.25 & -0.25 & -0.2 \\
$1.1 \%$ & -0.31 & -0.3 & -0.25 & -0.26 & 0 & -0.32 \\
$1.2 \%$ & -0.28 & -0.28 & -0.28 & -0.34 & -0.34 & -0.29 \\
$1.3 \%$ & -0.38 & -0.35 & -0.01 & -0.38 & -0.34 & -0.34 \\
$1.4 \%$ & -0.44 & -0.4 & -0.4 & -0.41 & -0.41 & -0.41 \\
\hline
\end{tabular}

In Table 3, we report the marginal nonlabor income effect estimates in the different specifications. We see that the estimated marginal effect is around -1 and fairly stable across specifications. In the specification with largest cross-validation value, the estimated marginal income effect is -1.02 The standard errors are around 0.13 and 0.14 throughout Table 3 .

Table 3: Estimated Income Effects 


\begin{tabular}{c|cccccc}
\hline Growth/Lambda & 2 & 1.5 & 1 & 0.5 & 0.2 & 0.1 \\
\hline $0 \%$ & -0.7 & -0.69 & -0.69 & -0.73 & -0.74 & 0.01 \\
$.1 \%$ & -0.85 & -0.79 & -0.79 & -0.93 & -0.87 & -0.09 \\
$.2 \%$ & -0.9 & -0.9 & -0.9 & -1 & -0.94 & -0.66 \\
$.3 \%$ & -0.99 & -0.9 & -0.9 & -1.02 & -1.09 & -1.12 \\
$.4 \%$ & -1.07 & -1.07 & -1.07 & -1.11 & -0.86 & -1.07 \\
$.5 \%$ & -1.14 & -1.14 & -1.14 & -1.17 & -1.18 & -0.96 \\
$.6 \%$ & -1.16 & -1.19 & -1.23 & -1.1 & -1.17 & -1.2 \\
$.7 \%$ & -1.22 & -1.23 & -1.15 & -1.18 & -1.19 & -1.26 \\
$.8 \%$ & -1.26 & -1.24 & -1.24 & -1.18 & -1.2 & -1.18 \\
$.9 \%$ & -1.15 & -1.15 & -1.15 & -1.17 & -1.23 & -1.17 \\
$1.0 \%$ & -1.22 & -1.23 & -1.23 & -1.15 & -1.16 & -1.15 \\
$1.1 \%$ & -1.2 & -1.21 & -1.11 & -1.13 & -1.49 & -1.23 \\
$1.2 \%$ & -1.09 & -1.09 & -1.09 & -1.2 & -1.2 & -1.11 \\
$1.3 \%$ & -1.1 & -1.11 & -1.37 & -1.14 & -1.08 & -1.08 \\
$1.4 \%$ & -1.01 & -1.04 & -1.04 & -1.06 & -1.06 & -1.06 \\
\hline
\end{tabular}

We also checked to see if the Slutzky condition of equation (6.8) was satisfied. We did this by plugging in estimated parameters in that equation and evaluating at $x_{c}$ and $y_{d}$ values given by marginal net-of-tax rate, marginal virtual income, and gross labor income quartiles, resulting in 27 inequalities for each specification. We found that each of these inequalities is satisfied until the growth rate is $0.5 \%$ The Slutzky conditions therefore hold in the specification with highest cross validation values and nearby specifications.

An income effect of -1 is larger in magnitude than found or assumed in most taxable income studies.Using similar data but a linear model Blomquist and Selin (2010) estimate a statistically significant negative income effect that is much closer to zero. Some studies just assume there is no income effect. It is important to account correctly for income effects in evaluating tax policy, because they have an impact on deadweight loss and other policy relevant variables. For example, by equation (2.10) of Auerbach (1985), the marginal deadweight loss in compensating variation terms is $(1-\theta)(y / \theta) e^{*}$ where $e^{*}$ is the compensated elasticity of taxable income. The compensated elasticity satisfies $e^{*}=e-\theta * \partial y / \partial R$ where $e$ is the uncompensated elasticity. Suppose that taxes were linear, the net of tax elasticity was .21, and the income effect -1 , corresponding to our estimates. In our data the net of tax rate is .32 on average. Thus, a compensated elasticity corresponding to our estimates is .53 $=.21-(.32) *(-1)$. This estimate is much larger than the uncompensated elasticity estimate of .21. It is also substantially larger than in Blomquist and Selin (2010), where a similar uncompensated elasticity but smaller income effect was estimated. Thus, allowing for general heterogeneity and accounting correctly for nonlinear taxes leads to a larger deadweight loss estimate through a larger income effect.

In Table 4 and 5, we investigate the influence of the productivity growth rate, covariates, instruments, and the regressor selection procedure on the estimates. We report net-of-tax 
elasticity (Table 4) and marginal income effect (Table 5) estimates from the highest crossvalidation values corresponding to productivity growth rates $.2, .3$, and .4 respectively. We also report specifications using a first- and a second-order power series approximation of the budget set. We start with specifications without demographic control variables and without instrumenting nonlabor income using the control function approach. We then add demographic control variables and finally also instrument nonlabor income by including the control function.

Table 4. Net-of-tax elasticity estimates by method

\begin{tabular}{r|rrrrr}
\hline & Lasso .2\% & Lasso .3\% & Lasso .4\% & First-order & Second-order \\
\hline No controls & 3.23 & 2.83 & 2.63 & 2.19 & 1.73 \\
& $(0.09)$ & $(0.09)$ & $(0.09)$ & $(0.07)$ & $(0.08)$ \\
Demo & 0.49 & 0.32 & 0.18 & 0.39 & 0.37 \\
& $(0.09)$ & $(0.09)$ & $(0.09)$ & $(0.07)$ & $(0.08)$ \\
Demo+IV & 0.38 & 0.21 & 0.07 & 0.32 & 0.27 \\
& $(0.09)$ & $(0.09)$ & $(0.09)$ & $(0.08)$ & $(0.08)$ \\
\hline
\end{tabular}

Table 5. Marginal income effects by method

\begin{tabular}{r|rrrrr}
\hline & Lasso .2\% & Lasso .3\% & Lasso .4\% & First-order & Second-order \\
\hline No controls & 0.04 & 0.12 & 0.04 & 0.04 & 0.19 \\
& $(0.00)$ & $(0.00)$ & $(0.00)$ & $(0.00)$ & $(0.00)$ \\
Demo & 0.03 & 0.09 & 0.03 & 0.03 & 0.13 \\
& $(0.00)$ & $(0.00)$ & $(0.00)$ & $(0.00)$ & $(0.00)$ \\
Demo+IV & -0.9 & -1.02 & -1.07 & -0.67 & -0.92 \\
& $(0.13)$ & $(0.13)$ & $(0.13)$ & $(0.13)$ & $(0.13)$ \\
\hline
\end{tabular}

We observe that accounting for demographics has a drastic effect on the estimated net-of-tax elasticity. This could occur because of high correlation of the budget set with background variables. Furthermore, instrumentation is crucial for obtaining negative marginal income effects. This is consistent with a positive correlation between different likely endogenous components of nonlabor income, such as capital income, and gross labor income. The specification using a first- or a second-order power series approximation produce elasticity and marginal effect estimates of the same magnitude as in the Lasso specifications.

We have also performed subsample estimation by splitting the sample by education level into a high- and a low-education subsample. In these estimations, we allow for different productivity growth in the two subsamples. Although estimated productivity growth differ slightly (higher for the high-education sample), the elasticities obtained are similar and not statistically significantly different from each other. We have experimented with alternative similar sample restrictions, definitions of labor income, and ways to handle the impact of the transfer system on the budget sets. The results are not sensitive to these issues. 


\subsection{Comparison with Linear Estimates}

To assess the importance of appropriately accounting for nonlinear budget set and productivity growth, we have estimated some different specifications based on linearizing the budget set at observed gross income levels. In these specifications, we regress gross labor income against the marginal net-of-tax rate and virtual income (the nonlabor income for the linearized budget set). To account for the endogeneity of the marginal net-of-tax rate to the budget set, we instrument it using the either the net-of-tax rate of the first segment or the last segment. To account for endogeneity of virtual income we instrument it using the same instrument as in the control variable estimates given above. We also control for county fixed effects, include year fixed effects, and the same covariates as used in nonparametric regression estimates above. We estimate specifications that are either linear or logarithmic in the gross labor income, marginal net-of-tax rate, and virtual income.

This type of specification is fairly common for taxable income estimation in repeated cross sections. The typical approach is, however, to use panel data like in Gruber and Saez (2002). This would allow for linear individual effects by differencing and enable us to use instruments based on lagged income. Note though that the typical approach does not allow for other individual heterogeneity, such as in the net of tax elasticity, while our estimates above allow for general individual heterogeneity. The linear specifications we consider here are meant only to provide a comparison with our nonlinear estimates but are not meant to be best possible estimates based on the typical linearization method.

The estimates based on instrumental variables linearization specifications are reported in Table 6. Standard errors constructed using the delta method are reported in parenthesis. The elasticities are evaluated at the sample mean income of 420,329 SEK. We report the same type of net-of-tax elasticity as before, but now for the linearized budget sets. We observe that the estimated elasticity is sensitive to the instrument used and functional form.

Table 6. Estimated elasticities using instrumental variables linearization specifications

\begin{tabular}{rr|cc}
\hline Instrument & Functional form & Net-of-tax elasticity & Income effect \\
\hline First segment & Linear & -.69 & -.92 \\
& & $(1.1)$ & $(.48)$ \\
First segment & Logarithmic & 4.3 & .44 \\
& & $(.18)$ & $(.061)$ \\
Last segment & Linear & 1.3 & .55 \\
& & $(0.30)$ & $(.28)$ \\
Last segment & Logarithmic & 1.0 & .075 \\
& & $(0.10)$ & $(.041)$ \\
\hline
\end{tabular}

In comparison with the nonparametric regression estimates using our method, we note that 
the estimated net-of-tax elasticity is much higher here while there are smaller income effects of opposite sign than previously. We find a statistically insignificant income effect when we use the log-log specification and instrument using the last segment tax rate. For our data, using our more sophisticated method therefore matters a lot. The overall picture is that correctly accounting for nonlinear budget sets and general heterogeneity is important in this data.

\subsection{Reform Estimates}

The expected value of taxable income is a useful tool for evaluating the effect of a tax reform on average taxable income. To illustrate this we use our estimated taxable income function to evaluate a tax program that was introduced in 2007 to 2008, which are the last two years of the sample period. In 2006, a center-right wing coalition government came into power in Sweden, and it launched a broader reform package to encourage labor supply among primarily low-income groups. The cornerstone of the package was an earned income tax credit (EITC) program that drastically lowered taxes on labor income. The credit was introduced in 2007 and reinforced in the subsequent three years. We investigate the effect of the 2008 version of the EITC on the labor supply of our sample individuals of married or cohabiting men using estimates from our preferred specifications.

The Swedish EITC program in 2008 is outlined in Table 7 for a representative individual. We observe that marginal tax rates were decreased quite much, by $16.40 \%$, in a small low income region between 23,200 and 54,300 SEK, and slightly, by $2.19 \%$, in a large medium income region between 148,900 and 431,600 SEK, where most married or cohabiting men are located. This construction creates positive substitution effects in these income ranges. However, it also creates income effects at most income levels. In particular, at higher income levels, there is only an income effect. For a sample of mostly full-time working individuals like ours, these income effects may be the most apparent effect on their labor supply. The Swedish EITC is different from the American and British equivalents by being universal, i.e., the same credit formula applies to everybody, and by not having a phase-out region where the credits received at lower income levels are phased out at higher income levels, creating stronger income effects.

Table 7. Swedish EITC program in 2008

\begin{tabular}{l|cc}
\hline Gross labor income & Net credits & Marginal tax rate change \\
\hline $0-23,200$ & 0 & 0 \\
$23,200-54,300$ & $(\text { Income- } 23,200)^{*} 0.1640$ & -0.164 \\
$54,300-148,900$ & 5,100 & 0 \\
$148,900-431,600$ & $5,100+(\text { Income-148,900 })^{*} 0.0219$ & -0.0219 \\
$>431,600$ & 11,300 & 0 \\
\hline
\end{tabular}

The estimated relative effects on gross labor income and government revenues are reported 
in Table 8. For gross labor income, we report the mean difference in the individual predicted income levels given their budget sets with (post-reform) and without (pre-reform) the EITC program respectively relative to the predicted income levels without the program (pre-reform). For tax revenues effects, we make the tax revenue predictions at the predicted income levels before calculating the mean relative effect in the same way as for gross labor income. We also report the average relative mechanical tax revenue effect in the absence of behavioral effects at predicted income levels without the program (pre-reform). Standard errors constructed using the delta method are reported for the reform effect on income in parenthesis.

Table 8. Estimated EITC effect on labor income and tax revenues.

\begin{tabular}{r|rrrrr}
\hline & Lasso $0.2 \%$ & Lasso $0.3 \%$ & Lasso $0.4 \%$ & First order & Second order \\
\hline Gross income & 3.8 & 2.86 & 1.96 & 0.69 & 1.29 \\
& $(0.45)$ & $(0.44)$ & $(0.43)$ & $(0.39)$ & $(0.4)$ \\
Tax revenues & 0.28 & -0.83 & -1.89 & -3.39 & -2.66 \\
Mechanical & -4.04 & -4.08 & -4.1 & -4.14 & -4.12 \\
\hline
\end{tabular}

We observe that the reform effect estimates vary with productivity growth rate as for the elasticity estimates in the Lasso selected specifications. The estimated reform effect on gross income decreases when productivity growth rate increases, which is a similar pattern as for the net-of-tax elasticity. At the specification with largest cross-validation values, where the productivity growth rate is $0.3 \%$, we obtain a statistically significant and positive reform effect on gross labor income of $2.86 \%$. However, the reform decreases tax revenues by $0.83 \%$. On the other hand, if there were no behavioral effects, tax revenues would have decreased by $4.08 \%$. The behavioral effects therefore reduce the cost of the reform by $80 \%$. The reform effect estimates on gross labor income are much smaller when using a first- or a second-order approximation of the budget set. This contrasts with the elasticity estimates where the Lasso specification produced similar results as linear and quadratic.

Two remarks are appropriate here. First, we include indirect taxation in our income and tax revenue measures. Revenues from these sources are larger than from direct labor taxes. Second, in nonlinear budget sets, tax revenues depend on the distribution of income under different tax regimes. In progressive tax systems, average revenues at predicted expected income levels are a downward biased estimate of expected revenues over the entire distribution. Because this bias may not be the same for the pre- and post-reform tax revenues, the reform effect tax revenue estimates may be biased.

\section{Conclusion}

In this paper we develop a method to nonparametrically estimate the expected value of taxable income as a function of a nonlinear budget set while allowing for general heterogeneity and 
optimization errors. We apply this approach to Swedish data and find a significant taxable income elasticity of .21 and an income effect of -1 . This income effect is more substantial than found in most taxable income studies.

This method could be extended to estimate the expected value of taxes. As with taxable income, for concave budget frontiers the expected value of taxes will depend only on the CDF of taxable income for a linear budget set. A straightforward calculation can be carried out to derive the expected value of taxes, which could then be estimated by a procedure analogous that the described above for taxable income.

\section{Appendix A: Proofs}

The following two technical conditions are referred to in the text and used in the proofs.

Assumption A1: $\eta$ belongs to a complete, separable metric space and $y(\rho, R, \eta), \partial y(\rho, R, \eta) / \partial \rho$, and $\partial y(\rho, R, \eta) / \partial R$ are continuous in $(\rho, R, \eta)$.

Assumption A2: $\eta=(u, \varepsilon)$ for scalar $\varepsilon$ and Assumption A1 is satisfied for $\eta=(u, \varepsilon)$ for a complete, separable metric space that is the product of a complete separable metric space for $u$ with Euclidean space for $\varepsilon, y(\rho, R, \eta)=y(\rho, R, u, \varepsilon)$ is continuously differentiable in $\varepsilon$, there is $C>0$ with $\partial y(\rho, R, u, \varepsilon) / \partial \varepsilon \geq 1 / C,\|\partial y(\rho, R, \eta) / \partial(\rho, R)\| \leq C$ everywhere, $\varepsilon$ is continuously distributed conditional on $u$, with conditional pdf $f_{\varepsilon}(\varepsilon \mid u)$ that is bounded and continuous in $\varepsilon$.

Before proving Theorem 1 we state a result on the derivatives of $F(y \mid \rho, R)$ with respect to $\rho$ and $R$.

Lemma A1: If Assumptions 1 and A2 are satisfied then $y(\rho, R, \eta)$ is continuously distributed for each $\rho, R>0$ and $F(y \mid \rho, R)$ is continuously differentiable in $y, \rho$, and $R$ and for the pdf $f_{y(\rho, R, \eta)}(y)$ of $y(\rho, R, \eta)$ at $y$,

$$
\begin{aligned}
& \frac{\partial F(y \mid \rho, R)}{\partial y}=f_{y(\rho, R, \eta)}(y) \\
& \frac{\partial F(y \mid \rho, R)}{\partial(\rho, R)}=-f_{y(\rho, R, \eta)}(y) E\left[\frac{\partial y(\rho, R, \eta)}{\partial(\rho, R)} \mid y(\rho, R, \eta)=y\right] .
\end{aligned}
$$

Proof: This follows exactly as in the proof of Lemma A1 of Hausman and Newey (2014). Q.E.D.

Proof of Theorem 1: Note that $y(\rho, R, \eta)$ is differentiable by Assumption 1. Also, $\rho$ behaves like the negative of a price (increasing $\rho$ increases utility), so that the Slutzky condition 
for taxable income is

$$
-\frac{\partial y(\rho, R, \eta)}{\partial \rho}+y(\rho, R, \eta) \frac{\partial y(\rho, R, \eta)}{\partial R} \leq 0 .
$$

By Assumption 1 and standard utility theory this inequality must be satisfied for all $\eta$ and all $\rho, R>0$. Then by Lemma A1 $F(y \mid \rho, R)$ is differentiable in $\rho$ and $R$ and

$$
\begin{aligned}
& \frac{\partial F(y \mid \rho, R)}{\partial \rho}-y \frac{\partial F(y \mid \rho, R)}{\partial R} \\
= & -f_{y(\rho, R, \eta)}(y)\left\{E\left[\frac{\partial y(\rho, R, \eta)}{\partial \rho} \mid y(\rho, R, \eta)=y\right]-y \cdot E\left[\frac{\partial y(\rho, R, \eta)}{\partial R} \mid y(\rho, R, \eta)=y\right]\right\} \\
= & f_{y(\rho, R, \eta)}(y) E\left[-\frac{\partial y(\rho, R, \eta)}{\partial \rho}+y(\rho, R, \eta) \frac{\partial y(\rho, R, \eta)}{\partial R} \mid y(\rho, R, \eta)=y\right] \leq 0,
\end{aligned}
$$

where the inequality follows by $f_{y(\rho, R, \eta)}(y) \geq 0$. This argument shows the first conclusion.

To show the second conclusion, for $0<\tau<1$ let $Q(\tau \mid \rho, R)=F^{-1}(\tau \mid \rho, R)$, which inverse function exists by $F(y \mid \rho, R)$ strictly increasing in $y$ on $\left(y_{\ell}, y_{u}\right)$ and $\left[y_{\ell}, y_{u}\right]$ being the support of $F(y \mid \rho, R)$. By the inverse function theorem, for all $\rho, R>0$,

$$
\begin{aligned}
& -\frac{\partial Q(\tau \mid \rho, R)}{\partial \rho}+Q(\tau \mid \rho, R) \frac{\partial Q(\tau \mid \rho, R)}{\partial R} \\
= & f_{y(\rho, R, \eta)}(Q(\tau \mid \rho, R))^{-1}\left\{\frac{\partial F(Q(\tau \mid \rho, R) \mid \rho, R)}{\partial \rho}-Q(\tau \mid \rho, R) \frac{\partial F(Q(\tau \mid \rho, R) \mid \rho, R)}{\partial R}\right\} \leq 0 .
\end{aligned}
$$

Therefore it follows by Hurwicz and Uzawa (1971) that for each $\tau$ with $0<\tau<1$ there is a utility function $U(c, y, \tau)$ with for all $\rho, R>0$,

$$
Q(\tau \mid \rho, R)=\arg \max _{c, y} U(c, y, \tau) \text { s.t. } \quad c=y \rho+R, c \geq 0, y \geq 0 .
$$

Let $\eta$ be distributed uniformly on $(0,1)$ and define

$$
y(\rho, R, \eta)=Q(\eta \mid \rho, R)
$$

Then

$$
\operatorname{Pr}(Q(\eta \mid \rho, R) \leq y)=\operatorname{Pr}(\eta \leq F(y \mid \rho, R))=F(y \mid \rho, R) .
$$

Thus, the RUM $U(c, y, \eta)$ has $F(y \mid \rho, R)$ as its CDF. Q.E.D.

Lemma A2: If Assumptions 1 and $A 1$ are satisfied and $B(y)$ is concave then $y(B, \eta)$ is unique and $U(B(y), y, \eta)$ is strictly increasing to the left of $y(B, \eta)$ and strictly decreasing to the right of $y(B, \eta)$.

Proof: For notational convenience suppress the $\eta$ argument, which is held fixed in this proof. Let $y^{*}=y(B)$. Suppose $y^{*}>0$. Consider $y<y^{*}$ and let $\tilde{y}$ such that $y<\tilde{y}<y^{*}$. Let $(\tilde{c}, \tilde{y})$ 
be on the line joining $(B(y), y)$ and $\left(B\left(y^{*}\right), y^{*}\right)$. By concavity of $B(\cdot), \tilde{c} \leq B(\tilde{y})$, so by strict quasi-concavity and the definition of $y^{*}$,

$$
U\left(B\left(y^{*}\right), y^{*}\right) \geq U(B(\tilde{y}), \tilde{y}) \geq U(\tilde{c}, \tilde{y})>\min \left\{U(B(y), y), U\left(B\left(y^{*}\right), y^{*}\right)\right\}=U(B(y), y) .
$$

Thus $U(B(\tilde{y}), \tilde{y})>U(B(y), y)$. An analogous argument gives $U(B(\tilde{y}), \tilde{y})>U(B(y), y)$ for $y>\tilde{y}>y^{*}$.Q.E.D.

Lemma A3: If Assumptions 1 and $A 1$ are satisfied and the budget frontier $B$ is concave then for each $y, \operatorname{Pr}(y(B, \eta) \leq y)=F(y \mid \rho(y), R(y))$.

Proof: Consider any fixed value of $y$ as in the statement of the Lemma and let $B(z)$ denote the value of the budget frontier for any value $z$ of taxable income. By concavity of $B(z)$ and Rockafellar (1970, pp. 214-215), $\rho(y)$ exists and is a subgradient of $B(z)$ at $y$. Define $\dot{\rho}=\rho(y)$ and $\dot{B}=B(y)$. For any $z$ let $\dot{B}(z)=\dot{B}+\dot{\rho}(z-y)=R(y)+\rho(y) z$ denote the linear budget frontier with slope $\rho(y)$ passing through $(B(y), y)$. Let $y^{*}=\operatorname{argmax}_{z} U(B(z), z)$ where we suppress the $\eta$ argument for convenience. Also let $\dot{y}^{*}=\arg \max _{z} U(\dot{B}(z), z)$. We now proceed to show that $y^{*} \leq y \Longleftrightarrow \dot{y}^{*} \leq y$.

It follows by $\dot{\rho}$ being a subgradient at $y$ of $B(z)$ and $B(z)$ being concave that for all $z$,

$$
\dot{B}(z) \geq B(z)
$$

Therefore $\dot{B}\left(y^{*}\right) \geq B\left(y^{*}\right)$, so that

$$
U\left(\dot{B}\left(y^{*}\right), y^{*}\right) \geq U\left(B\left(y^{*}\right), y^{*}\right) \geq U(B(y), y)=U(\dot{B}(y), y)
$$

Note that $\dot{B}(z)$ is linear and hence concave so that by Lemma A2, $U(\dot{B}(z), z)$ is strictly increasing to the left of $\dot{y}^{*}$ and decreasing to the right of $\dot{y}^{*}$. Suppose that $y<y^{*}$. Then $y<\dot{y}^{*}$, because otherwise $\dot{y}^{*} \leq y<y^{*}$ and the above equation contradicts that $U(\dot{B}(z), z)$ is strictly decreasing to the right of $\dot{y}^{*}$. Similarly, if $y>y^{*}$ then $y>\dot{y}^{*}$, because otherwise $\dot{y}^{*} \geq y>y^{*}$ and the above equation contradicts that $U(\dot{B}(z), z)$ is strictly increasing to the left of $\dot{y}^{*}$.

Next suppose $y^{*}=y$. Let $\ddot{\rho}$ be the slope of a line that separates the set weakly preferred to $(B(y), y)$ and the budget set and let $\ddot{B}(z)=\dot{B}+\ddot{\rho}\left(z-y^{*}\right)$, so that $U\left(\dot{B}, y^{*}\right) \geq U(\ddot{B}(z), z)$ for all $z$. Then by Lemma A2 applied to the budget frontier $\ddot{B}(z), U\left(\dot{B}, y^{*}\right)>U(\ddot{B}(z), z)$ for all $\tilde{y} \neq y^{*}$. Also, by Rockafellar (1970, pp. 214-215) $\ddot{\rho} \geq \dot{\rho}$. Then for any $z>y^{*}$ we have

$$
\dot{B}+\ddot{\rho}\left(z-y^{*}\right) \geq \dot{B}+\dot{\rho}\left(z-y^{*}\right)=\dot{B}(z) .
$$

so that

$$
U(\dot{B}(z), z) \leq U\left(\dot{B}+\ddot{\rho}\left(z-y^{*}\right), z\right)=U(\ddot{B}(z), z)<U\left(\dot{B}, y^{*}\right)
$$


It follows that $\dot{y}^{*} \leq y^{*}=y$. Thus, we have show that $y^{*}=y$ implies $\dot{y}^{*} \leq y$. Together with the implication of the previous paragraph this means that $y^{*} \leq y \Longrightarrow \dot{y}^{*} \leq y$.

Summarizing, we have shown that

$$
y^{*} \leq y \Longrightarrow \dot{y}^{*} \leq y \text { and } y^{*}>y \Longrightarrow \dot{y}^{*}>y \text {. }
$$

Therefore $y^{*} \leq y \Longleftrightarrow \dot{y}^{*} \leq y$.

Note that $y^{*}$ is the utility maximizing point on the budget frontier $B(z)$ while $\dot{y}^{*}$ is the utility maximizing point on the linear budget frontier $\dot{B}(z)=B(y)+\rho(y)(z-y)=R(y)+\rho(y) z$. Thus, $y^{*} \leq y \Longleftrightarrow \dot{y}^{*} \leq y$ means that the event $y(B, \eta) \leq y$ coincides with the event that $\arg \max _{z} U(R(y)+\rho(y) z, z, \eta) \leq y$, i.e. with the event the optimum on the linear budget set is less than or equal to $y$. The probability that the optimum on this linear budget is less than or equal to $y$ is $F(y \mid \rho(y), R(y))$, giving the conclusion. Q.E.D.

Lemma A4: If Assumptions 1 and $A 1$ are satisfied then for all y such that there is $\Delta>0$ with $\bar{B}(z)=B(z)$ for $z \in[y, y+\Delta]$ we have $\operatorname{Pr}(y(B, \eta) \leq y)=\operatorname{Pr}(y(\bar{B}, \eta) \leq y)$.

Proof: Note that $\bar{B}(z) \geq B(z)$ for all $z$. For notational simplicity suppress the $\eta$ argument and let $U(c, y)=U(c, y, \eta)$. Let

$$
y^{*} \stackrel{\text { def }}{=} \operatorname{argmax}_{z} U(B(z), z), \bar{y}^{*} \stackrel{\text { def }}{=} \operatorname{argmax}_{z} U(\bar{B}(z), z) \text {. }
$$

Suppose first that $y^{*}=y$. Then for any $z \in(y, y+\Delta]$,

$$
U(\bar{B}(y), y)=U(B(y), y) \geq U(B(z), z)=U(\bar{B}(z), z) .
$$

By Lemma A2 we cannot have $\bar{y}^{*}>y$ because then the above inequality is not consistent with $U(\bar{B}(z), z)$ being strictly monotonically increasing to the left of $\bar{y}^{*}$. Therefore $\bar{y}^{*} \leq y$. Suppose next that $y^{*}<y$. Then

$$
U\left(\bar{B}\left(y^{*}\right), y^{*}\right) \geq U\left(B\left(y^{*}\right), y^{*}\right) \geq U(B(y), y)=U(\bar{B}(y), y)
$$

Then by similar reasoning as before $\bar{y}^{*} \leq y$. Thus we have now show that

$$
y^{*} \leq y \Longrightarrow \bar{y}^{*} \leq y \text {. }
$$

Next, suppose that $y^{*}>y$. Then there is $z \in(y, y+\Delta]$ with $y<z<y^{*}$

$$
U\left(\bar{B}\left(y^{*}\right), y^{*}\right) \geq U\left(B\left(y^{*}\right), y^{*}\right) \geq U(B(z), z)=U(\bar{B}(z), z) .
$$


Again by Lemma A2 we cannot have $\bar{y}^{*} \leq y$ because then $\bar{y}^{*}<z<y^{*}$ and the above inequality is not consistent with $U(\bar{B}(z), z)$ being strictly monotonic decreasing to the right of $\bar{y}^{*}$. Therefore, $\bar{y}^{*}>y$, and we have shown that

$$
y^{*}>y \Longrightarrow \bar{y}^{*}>y \text {. }
$$

Therefore we have $y^{*} \leq y \Longleftrightarrow \bar{y}^{*} \leq y$, so the conclusion follows similarly to the conclusion of Lemma A2. Q.E.D.

Proof of Theorem 2: Combining the conclusions of Lemmas A3 and A4 gives

$$
\operatorname{Pr}(y(B, \eta) \leq y)=\operatorname{Pr}(y(\bar{B}, \eta) \leq y)=F(y \mid \rho(y), R(y)) \text {.Q.E.D. }
$$

Proof of Theorem 3: For notational simplicity we suppress the $\eta$ as before. Let $\hat{y}=$ $y(B), \check{y}=\bar{y}(B)$ denote the lower and upper bound respectively. Let $\tilde{B}(B)=\{(c, y): c \leq$ $B(y), \hat{y} \leq y \leq \check{y}\}, y^{*}=y(B, \eta)$, and consider

$$
\tilde{y}^{*}=\arg \max _{z} U(B(z), z) \text { s.t. } \hat{y} \leq z \leq \check{y}
$$

By construction, for all $y$

$$
F_{\tilde{y}(B, \eta)}(y \mid B)=F_{\tilde{y}(B, \eta)}(y \mid \tilde{B}) .
$$

Now consider any $y \in[\hat{y}, \check{y})$. We will prove that $y^{*} \leq y$ if and only if $\tilde{y} \leq y$.

Suppose first that $y^{*} \leq y$. If $y^{*} \geq \hat{y}$ then $y^{*}=\tilde{y}^{*}$, so that $\tilde{y}^{*} \leq y$. If $y^{*}<\hat{y}$, then by Lemma A1, for all $z \in(\hat{y}, \check{y}]$ and the upper boundary $\bar{B}(z)$ of the convex hull $\bar{B}$ of $B$,

$$
U\left(B\left(y^{*}\right), y^{*}\right)>U(B(\hat{y}), \hat{y})>U(\bar{B}(z), z) \geq U(B(z), z),
$$

so that $\tilde{y}^{*}=\hat{y} \leq y$. Therefore, $y^{*} \leq y \Longrightarrow \tilde{y}^{*} \leq y$.

Next, suppose that $y^{*}>y$. If $y^{*} \leq \check{y}$ then $\tilde{y}^{*}=y^{*}$ so that $\tilde{y}^{*}>y$. Suppose $y^{*}>\check{y}$. Then by Lemma A1 and $y<\check{y}$, for all $z \in[\hat{y}, \check{y})$ we have

$$
U\left(B\left(y^{*}\right), y^{*}\right)>U(B(\check{y}), \check{y})>U(\bar{B}(z), z) \geq U(B(z), z)
$$

so that $\tilde{y}^{*}=\check{y}>y$. Therefore $y^{*}>y \Longrightarrow \tilde{y}^{*}>y$.

Summarizing, for $y \in[\hat{y}, \check{y})$ we have $y^{*} \leq y \Longleftrightarrow \tilde{y}^{*} \leq y$. Therefore, adding back the $\eta$ notation, we have

$$
F_{y(B, \eta)}(y \mid B)=F_{\tilde{y}(B, \eta)}(y \mid B)=F_{\tilde{y}(B, \eta)}(y \mid \tilde{B}) \cdot Q \cdot E \cdot D .
$$

Proof of Theorem 4: Let $F(y)$ be the CDF of $y(B, \eta)$. By standard probability theory, $\Pi_{\bar{\ell}}=F(\bar{\ell})-\lim _{y \uparrow \bar{\ell}} F(y)$. By Theorem $2 F(\bar{\ell})=F\left(\bar{\ell} \mid \rho_{+}, R_{+}\right)$and $\lim _{y \uparrow \bar{\ell}} F(y)=\lim _{y \uparrow \bar{\ell}} F\left(y \mid \rho_{-}, R_{-}\right)$. 
Furthermore, by Assumption A2 $y\left(\rho_{-}, R_{-}, \eta\right)$ is continuously distributed so that $\lim _{y \uparrow \bar{\ell}} F\left(y \mid \rho_{-}, R_{-}\right)=$ $F\left(\bar{\ell} \mid \rho_{-}, R_{-}\right)$. Define $\Lambda(\rho)=F(\bar{\ell} \mid \rho, R(\rho))$ for $\rho \in\left[\rho_{+}, \rho_{-}\right]$. We then have

$$
\Pi_{\bar{\ell}}=\Lambda\left(\rho_{+}\right)-\Lambda\left(\rho_{-}\right) .
$$

By the chain rule, $R(\rho)=R_{-}+\bar{\ell}\left(\rho_{-}-\rho\right)$, and Lemma A1 of Hausman and Newey $(2014), \Lambda(\rho)$ is differentiable and

$$
\frac{d \Lambda(\rho)}{d \rho}=F_{\rho}(\bar{\ell} \mid \rho, R(\rho))-\bar{\ell} F_{R}(\bar{\ell} \mid \rho, R(\rho))=-\phi(\rho) \delta(\rho) .
$$

The conclusion then follows by the fundamental theorem of calculus. Q.E.D.

Proof of Theorem 5: Note that $E[Y]=E[y(B, \eta)]$ by hypothesis. Let $F_{j}(y)=F\left(y \mid \rho_{j}, R_{j}\right)$. By Theorem 2 , the CDF of $y(B, \eta)$ on $\left(\ell_{j-1}, \ell_{j}\right)$ is $F_{j}(y)$. Therefore,

$$
E[Y]=\sum_{j=1}^{J-1}\left[\int 1\left(\ell_{j-1}<y<\ell_{j}\right) y F_{j}(d y)+\ell_{j} \operatorname{Pr}\left(Y(B, \eta)=\ell_{j}\right)\right]+\int 1\left(\ell_{J-1}<y\right) y F_{J}(d y) .
$$

Note that

$$
\int 1\left(\ell_{J-1}<y\right) y F_{J}(d y)=\bar{y}\left(\rho_{J}, R_{J}\right)-\int 1\left(y \leq \ell_{J-1}\right) y F_{J}(d y) .
$$

In addition, by $\ell_{0}=0$ we have $\int 1\left(y \leq \ell_{0}\right) y F_{1}(d y)=0$, so that

$$
\begin{aligned}
\sum_{j=1}^{J-1} \int 1\left(\ell_{j-1}\right. & \left.<y<\ell_{j}\right) y F_{j}(d y)+\int 1\left(\ell_{J-1}<y\right) y F_{J}(d y) \\
& =\sum_{j=1}^{J-1} \int\left[1\left(y<\ell_{j}\right)-1\left(y \leq \ell_{j-1}\right)\right] y F_{j}(d y)+\int 1\left(\ell_{J-1}<y\right) y F_{J}(d y) \\
& =\bar{y}\left(\rho_{J}, R_{J}\right)+\sum_{j=1}^{J-1}\left[\int 1\left(y<\ell_{j}\right) y F_{j}(d y)-\int 1\left(y \leq \ell_{j}\right) y F_{j+1}(d y)\right]
\end{aligned}
$$

Also, it follows from Theorem 2, similarly to the proof of Theorem 4, that

$$
\begin{aligned}
\operatorname{Pr}(y(B, \eta) & \left.=\ell_{j}\right)=F_{j+1}\left(\ell_{j}\right)-\lim _{y \uparrow \ell_{j}} F_{j}(y)=\int 1\left(y \leq \ell_{j}\right) F_{j+1}(d y)-\int 1\left(y<\ell_{j}\right) F_{j}((d, j) .11) \\
& =\int 1\left(y \geq \ell_{j}\right) F_{j}(d y)-\int 1\left(y>\ell_{j}\right) F_{j+1}(d y) .
\end{aligned}
$$

Combining these results we have

$$
E[Y]=\bar{y}\left(\rho_{J}, R_{J}\right)+\sum_{j=1}^{J-1}\left[\int 1\left(y<\ell_{j}\right)\left(y-\ell_{j}\right) F_{j}(d y)-\int 1\left(y \leq \ell_{j}\right)\left(y-\ell_{j}\right) F_{j+1}(d y)\right] .
$$

Noting that $\int 1\left(y \leq \ell_{j}\right)\left(y-\ell_{j}\right) F_{j+1}(d y)=\int 1\left(y<\ell_{j}\right)\left(y-\ell_{j}\right) F_{j+1}(d y)$ then gives the first conclusion. 
To show the second conclusion, note that by $\ell_{0}=0$ we have $\bar{y}\left(\rho_{1}, R_{1}\right)=\int 1\left(y>\ell_{0}\right) y F_{1}(d y)$. Then it follows that

$$
\begin{aligned}
\sum_{j=1}^{J-1} \int 1\left(\ell_{j-1}\right. & \left.<y<\ell_{j}\right) y F_{j}(d y)+\int 1\left(\ell_{J-1}<y\right) y F_{J}(d y) \\
& =\sum_{j=1}^{J-1} \int\left\{1\left(y>\ell_{j-1}\right)-1\left(y \geq \ell_{j}\right)\right\} y F_{j}(d y)+\int 1\left(y>\ell_{J-1}\right) y F_{J}(d y) \\
& =\bar{y}\left(\rho_{1}, R_{1}\right)+\sum_{j=1}^{J-1}\left[\int 1\left(y>\ell_{j}\right) y F_{j+1}(d y)-\int 1\left(y \geq \ell_{j}\right) y F_{j}(d y)\right] .
\end{aligned}
$$

Combining this with the second equality in eq. (11.11) then gives

$$
E[Y]=\bar{y}\left(\rho_{1}, R_{1}\right)+\sum_{j=1}^{J-1}\left[\int 1\left(y>\ell_{j}\right)\left(y-\ell_{j}\right) F_{j+1}(d y)-\int 1\left(y \geq \ell_{j}\right)\left(y-\ell_{j}\right) F_{j}(d y)\right] .
$$

Noting that $\int 1\left(y \geq \ell_{j}\right)\left(y-\ell_{j}\right) F_{j}(d y)=\int 1\left(y>\ell_{j}\right)\left(y-\ell_{j}\right) F_{j}(d y)$ then gives the second conclusion. Q.E.D.

Proof of Theorem 6: By Edmunds and Evans (1989) there exists $C$ such that for each $A$ and $B$ there is $\left(\beta_{a b}\right)$ such that for $\varepsilon=C A^{1-s} B^{(1-s) / 2}$ and $p^{A B}(y, x)=\sum_{a=1}^{A} \sum_{b=1}^{B} \beta_{a b} f_{y}(y) r_{b}(x)$,

$$
\sup _{\mathcal{Z}}\left|f(y \mid x)-p^{A B}(y, x)\right|+\sup _{\mathcal{Z}}\left|f_{\rho}(y \mid x)-p_{\rho}^{A B}(y, x)\right|+\sup _{\mathcal{Z}}\left|f_{R}(y \mid x)-p_{R}^{A B}(y, x)\right| \leq \varepsilon,
$$

where the subscripts denote partial derivatives and $\mathcal{Z}=\mathcal{Y} \times \mathcal{X}$. Let

$$
\tilde{p}^{A B}(y, x)=f_{1}(y)+\sum_{a=2}^{A} w_{a}(x, \beta)\left[f_{a}(y)-f_{1}(y)\right]=p^{A B}(y, x)+\left[1-\sum_{a=1}^{A} w_{a}(x, \beta)\right] f_{1}(y) .
$$

Note that by $\int f_{a}(y) d y=1$ for each $a$ and by $\mathcal{Y}$ bounded,

$$
\sup _{\mathcal{X}}\left|1-\sum_{a=1}^{A} w_{a}(x, \beta)\right|=\sup _{\mathcal{X}}\left|\int\left[f(y \mid x)-p^{A B}(y, x)\right] d y\right| \leq \sup _{\mathcal{X}} \int\left|f(y \mid x)-p^{A B}(y, x)\right| d y \leq C \varepsilon .
$$

Also,

$\sup _{\mathcal{X}}\left|\frac{\partial}{\partial \rho} \sum_{a=1}^{A} w_{a}(x, \beta)\right|=\sup _{\mathcal{X}}\left|\frac{\partial}{\partial \rho} \int\left[f(y \mid x)-p^{A B}(y, x)\right] d y\right| \leq \sup _{\mathcal{X}} \int\left|f_{\rho}(y \mid x)-p_{\rho}^{A B}(y, x)\right| d y \leq C \varepsilon$,

and $\sup _{\mathcal{X}}\left|\frac{\partial}{\partial R} \sum_{a=1}^{A} w_{a}(x, \beta)\right| \leq C \varepsilon$ similarly. Recall that $\bar{y}(x)=\int y f(y \mid x) d y$ and $\nu(x, \ell)=$ $\int 1(y<\ell)(y-\ell) f(y \mid x) d y$. Define

$$
\bar{y}^{A B}(x)=\int y \cdot \tilde{p}^{A B}(y, x) d y, \nu^{A B}(x, \ell)=\int 1(y<\ell)(y-\ell) \tilde{p}^{A B}(y, x) d y .
$$


Note that for any pdf $f_{1}(y), \int y f_{1}(y) d y \leq \sup _{\mathcal{Y}} y=C$, so for all $x \in \mathcal{X}$,

$$
\begin{aligned}
\left|\bar{y}(x)-\bar{y}^{A B}(x)\right| & =\left|\int y\left[f(y \mid x)-p^{A B}(y, x)\right] d y+\left[1-\sum_{a=1}^{A} w_{a}(x, \beta)\right] \int y f_{1}(y) d y\right| \\
& \leq \int y\left|f(y \mid x)-p^{A B}(y, x)\right| d y+\left|1-\sum_{a=1}^{A} w_{a}(x, \beta)\right| \int y f_{1}(y) d y \leq C \varepsilon .
\end{aligned}
$$

Let $\Delta(x, \ell)=\nu(x, \ell)-\nu^{A B}(x, \ell)$. Note that by $\mathcal{Y}$ bounded there is $C$ such that for all $\ell \in \mathcal{Y}$ and any pdf $f_{1}(y)$ with support contained in $\mathcal{Y},\left|\int 1(y<\ell)(y-\ell) f_{1}(y) d y\right| \leq C$. Therefore,

$$
\begin{aligned}
\left|\frac{\partial}{\partial \rho} \Delta(x, \ell)\right| & =\mid \frac{\partial}{\partial \rho} \int 1(y<\ell)(y-\ell)\left[f(y \mid x)-p^{A B}(y, x)\right] d y+\frac{\partial}{\partial \rho}\left[1-\sum_{a=1}^{A} w_{a}(x, \beta)\right] \int 1(y<\ell)(y-\ell) f_{1}(y) d y \\
& \leq C \varepsilon
\end{aligned}
$$

Therefore we have

$$
\begin{aligned}
& \left|\sum_{j=1}^{J-1}\left[\nu\left(x_{j}, \ell_{j}\right)-\nu\left(x_{j+1}, \ell_{j}\right)\right]-\sum_{j=1}^{J-1}\left[\nu^{A B}\left(x_{j}, \ell_{j}\right)-\nu^{A B}\left(x_{j+1}, \ell_{j}\right)\right]\right| \\
= & \left.\left|\sum_{j=1}^{J-1}\left[\Delta\left(x_{j}, \ell_{j}\right)-\Delta\left(x_{j+1}, \ell_{j}\right)\right]\right|=\mid \sum_{j=1}^{J-1}\left[\frac{\partial \Delta\left(\bar{x}_{j}, \ell_{j}\right)}{\partial x}\right]^{T}\left(x_{j}-x_{j+1}\right)\right] \mid \\
\leq & C\left(\sup _{\mathcal{X}}\left|\frac{\partial \Delta\left(\bar{x}_{j}, \ell_{j}\right)}{\partial \rho}\right|+\sup _{\mathcal{X}}\left|\frac{\partial \Delta\left(\bar{x}_{j}, \ell_{j}\right)}{\partial R}\right|\right) \sum_{j=1}^{J-1}\left[\rho_{j}-\rho_{j+1}+R_{j+1}-R_{j}\right] \\
\leq & C \varepsilon\left[\rho_{1}-\rho_{J}+R_{J}-R_{1}\right] \leq C \varepsilon .
\end{aligned}
$$

To conclude the proof, note that

$$
\begin{aligned}
\sum_{j=1}^{J-1}\left[\nu^{A B}\left(x_{j}, \ell_{j}\right)-\nu^{A B}\left(x_{j+1}, \ell_{j}\right)\right] & =\sum_{j=1}^{J-1}\left[\int 1\left(y<\ell_{j}\right)\left(y-\ell_{j}\right)\left[p^{A B}\left(y, x_{j}\right)-p^{A B}\left(y, x_{j+1}\right)\right] d y\right. \\
& =\sum_{j=1}^{J-1}\left[\int 1\left(y<\ell_{j}\right)\left(y-\ell_{j}\right) \sum_{a=2}^{A}\left[w_{a}\left(x_{j}, \beta\right)-w_{a}\left(x_{j+1}, \beta\right)\right]\left[f_{a}(y)-f_{1}(y)\right] d y\right. \\
& =\sum_{a=2}^{A} \sum_{j=1}^{J-1}\left[w_{a}\left(x_{j}, \beta\right)-w_{a}\left(x_{j+1}, \beta\right)\right]\left[\nu_{a}\left(\ell_{j}\right)-\nu_{1}\left(\ell_{j}\right)\right] \\
& =\sum_{a=2}^{A} \sum_{b=1}^{B} \beta_{a b} \sum_{j=1}^{J}\left[r_{b}\left(x_{j}\right)-r_{b}\left(x_{j+1}\right)\right]\left[\nu_{a}\left(\ell_{j}\right)-\nu_{1}\left(\ell_{j}\right)\right] .
\end{aligned}
$$

From these two equations we see that the expression in the statement of the theorem is

$$
E[Y]-\bar{y}^{A B}\left(x_{J}\right)-\sum_{j=1}^{J-1}\left[\nu^{A B}\left(x_{j}, \ell_{j}\right)-\nu^{A B}\left(x_{j+1}, \ell_{j}\right)\right] .
$$

The conclusion then follows by the triangle inequality, Theorem 5 , and the above bonds on $\left|\bar{y}(x)-\bar{y}^{A B}(x)\right|$ and $\left|\sum_{j=1}^{J-1}\left[\nu\left(x_{j}, \ell_{j}\right)-\nu\left(x_{j+1}, \ell_{j}\right)\right]-\sum_{j=1}^{J-1}\left[\nu^{A B}\left(x_{j}, \ell_{j}\right)-\nu^{A B}\left(x_{j+1}, \ell_{j}\right)\right]\right|$. Q.E.D. 


\section{Appendix B: Sample Statistics Sweden}

$\begin{array}{llllll}\text { Variable } & \text { All years } & 1993 & 1998 & 2003 & 2008 \\ \text { Number of observations } & 78,268 & 5,003 & 4,244 & 5,403 & 4,915 \\ \text { Gross labor income } & 4.18 & 3.79 & 4.11 & 4.23 & 4.62 \\ \text { 1-st net-of-tax rate } & 0.52 & 0.54 & 0.50 & 0.55 & 0.53 \\ \text { 1-st virtual income } & 1.86 & 1.76 & 1.77 & 1.73 & 1.69 \\ \text { Marginal net-of tax-rate } & 0.32 & 0.32 & 0.29 & 0.33 & 0.33 \\ \text { Marginal virtual income } & 2.17 & 2.07 & 2.08 & 2.03 & 2.09 \\ \text { Last net-of-tax rate } & 0.25 & 0.29 & 0.25 & 0.25 & 0.24 \\ \text { Last virtual income } & 2.42 & 2.15 & 2.20 & 2.38 & 2.52 \\ \text { Age } & 43.80 & 42.26 & 43.77 & 44.23 & 44.23 \\ \text { Dummy children < 6 years } & 0.35 & 0.44 & 0.36 & 0.30 & 0.33 \\ \text { Dummy foreign born } & 0.13 & 0.10 & 0.12 & 0.14 & 0.17 \\ \text { Wife's net labor income } & 1.45 & 1.25 & 1.28 & 1.54 & 1.86\end{array}$

Notes: Gross labor income, wife's net labor income, and virtual incomes are expressed in 100,000 SEK.

\section{References}

Aaberge, R. and L. Flood (2008), "Evaluation of an In-work Tax Credit Reform in Sweden: Effects on Labor Supply and Welfare Participation of Single Mothers," IZA Discussion Paper 3736 .

Auerbach, A. (1985), "The Theory of Excess Burden and Optimal Taxation," in A. Auerbach and M. Feldstein, eds., Handbook of Public Economics Vol. 1, 61-128.

Auten, G. and R. Carroll (1999), "The Effect of Taxes on Household Income," Review of Economics and Statistics 81: 681-693.

Belloni, A. and V. Chernozhukov (2013): "Least Squares After Model Selection in HighDimensional Sparse Models," Bernoulli , 521-547.

Blomquist, S. and W. Newey (2002), "Nonparametric Estimation with Nonlinear Budget Sets," Econometrica 70(6): 2455-2480.

Blomquist, S. and H. Selin (2010), "Hourly Wage Rate and Taxable Labor Income Responsiveness to Changes in Marginal Tax Rates," Journal of Public Economics 94: 878-889.

Blundell, R. and R. Matzkin (2014), "Control Functions in Nonseparable Simultaneous Equations Models," Quantitative Economics 5, 271-295.

Blundell R.W. and J.L. Powell (2006), "Endogeneity in Nonparametric and Semiparametric Regression Models," in Dewatripont, M., L.P. Hansen, and S.J. Turnovsky, eds., Advances 
in Economics and Econometrics: Theory and Applications, Eighth World Congress, Vol. II, Cambridge University Press.

Blundell, R. and A. Shephard (2012), "Employment, hours of work and the optimal taxation of low-income families," Review of Economic Studies 79, 481-510.

Burtless, G. and J. Hausman (1978), "The Effect of Taxation on Labor Supply: Evaluating the Gary Negative Income Tax Experiment," Journal of Political Economy 86: 1103-1130.

Carroll, R. (1998), "Do Taxpayers Really Respond to Changes in Tax Rates? Evidence from the 1993 Tax Act," Office of Tax Analysis Working Paper No. 78., Washington, D.C.: U.S. Department of Treasury.

Chetty, R. (2012), "Bounds on Elasticities With Optimization Frictions: A Synthesis of Micro and Macro Evidence on Labor Supply," Econometrica 80, 969-1018.

Dette, H., S. Hoderlein, and N. Nuemeyer (2011) Testing Multivariate Economic Restrictions Using Quantiles: The Example of Slutsky Negative Semidefiniteness, working paper, Boston College.

Edmunds, D. E., and W. Evans (1989): "Entropy Numbers and Approximation Numbers in Function Spaces," Proceedings of the London Mathematical Society 58, 137-152.

Eissa, N. and S. Giertz (2006), "Trends in High Incomes and Behavioural Responses to Taxation: Evidence from Executive Compensation and Statistics of Income Data," Congressional Budget Office Working Paper No. 2006-14.

Ericson, P., R. Wahlberg, and L. Flood (2009), "SWEtaxben: A Swedish Tax/Benefit Micro Simulation Model and an Evaluation of a Swedish Tax Reform," IZA Discussion Paper 4106.

Feldstein, M. (1995), "The Effects of Marginal Tax Rates on Taxable Income: A Panel Study of the 1986 Tax Reform Act," Journal of Political Economy 103(3): 551 -572.

Feldstein, M. (1999), "Tax Avoidance and the Deadweight Loss of the Income Tax," Review of Economics and Statistics 81(4): 674-680.

Feldstein, M. and D. Feenberg, D. (1995), "The Effects of Increased Tax Rates on Taxable Income and Economic Efficiency: A Preliminary Analysis of the 1993 Tax Rate Increases," NBER Working Paper No. W5370.

Flood, L., R. Wahlberg, and E. Pylkkänen, (2007), "From Welfare to Work: Evaluating a Tax and Benefit Reform Targeted at Single Mothers in Sweden," Labour 21: 443-471.

Giertz, S. (2007), "The Elasticity of Taxable Income Over the 1980s and 1990s," National Tax Journal 60(4): 743-768.

Goolsbee, A. (1999), "Evidence on the High-Income Laffer Curve from Six Decades of Tax Reform," Brookings Papers on Economic Activity, No. 2 (1999), 1-64.

Gruber, J. and E. Saez (2002), "The Elasticity of Taxable Income: Evidence and Implica- 
tions," Journal of Public Economics 84:1-32.

Hausman, J. and W. Newey (2014), "Individual Heterogeneity and Average Welfare", working paper.

Hurwicz, L. and H. Uzawa (1971). "On the Integrability of Demand Functions," in Chipman, L. Hurwicz, M. K. Richter, and H. F. Sonnenschein, eds. 1971. Preferences, Utility, and Demand: A Minnesota Symposium. New York: Harcourt, Brace, Jovanovich, 114-148.

Keane, M.P. (2011). "Labor Supply and Taxes: A Survey," Journal of Economic Literature 49, 961-1075.

Keane, M. and R. Moffitt (1998): "A Structural Model of Multiple Welfare Program Participation and Labor Supply," International Economic Review 39, 553-589.

Kleven, H. J., M. Knudsen, C.T. Kreiner, S. Pedersen and E. Saez (2011), "Unwilling or Unable to Cheat? Evidence from a Randomized Tax Audit Experiment in Denmark," Econometrica 79: 651-692.

Kopczuk, W. (2005), "Tax Bases, Tax Rates and the Elasticity of Taxable Income," Journal of Public Economics 89(11-12): 2093-2119.

Lindsey, L. (1987), "Individual Taxpayer Response to Tax Cuts: 1982-1984, with Implications for the Revenue Maximizing Tax Rate," Journal of Public Economics 33: 173-206.

Looney, A. and M. Singhal (2006), "The Effect of Anticipated Tax Changes on Intertemporal Labor Supply and the Realization of Taxable Income," NBER Working Paper 12417, National Bureau of Economic Research.

Manski, C. (2013) "Identification of Income-Leisure Preferences and Evaluation of Income Tax Policy," Quantitative Economics, forthcoming.

McFadden, D. (2005) "Revealed Stochastic Preference: A Synthesis," Economic Theory 26, 245-264.

McFadden, D. and K. Richter (1991): "Stochastic Rationality and Revealed Stochastic Preference," in J. Chipman, D. McFadden, K. Richter (eds) Preferences, Uncertainty, and Rationality, Westview Press, 161-186.

Moffitt, R. and M. Wilhelm (2000), "Taxation and the Labor Supply Decisions of the Affluent" in Does Atlas Shrug? The Economic Consequences of Taxing the Rich, J. Slemrod (ed.), New York: Cambridge University Press.

Navratil, J. (1995), "The Economic Recovery Tax Act of 1981: Evidence on Individual Taxpayer Behavior from Panel Tax Return Data," PhD thesis, Department of Economics, Harvard University.

Rockafellar, R.T. (1970) Convex Analysis, Princeton, Princeton University Press.

Saez, E. (2003), "The Effect of Marginal Tax Rates on Income: A Panel Study of 'Bracket 
Creep'," Journal of Public Economics 85: 1231-1258.

Saez, E. (2010), "Do Taxpayers Bunch at Kink Points," American Economic Journal: Economic Policy 2, 180-212.

Saez, E., J. Slemrod, and S. Giertz (2012), "The Elasticity of Taxable Income with Respect to Marginal Tax Rates: A Critical Review," Journal of Economic Literature 50: 3-50.

Sammartino, F. and D. Weiner (1997), "Recent Evidence on Taxpayers' Response to the Rate Increases in the 1990's," National Tax Journal 50(3): 683-705.

Sillamaa, M. and M. Veall (2000), "The Effect of Marginal Tax Rates on Taxable Income: A Panel Study of the 1988 Tax Flattening in Canada," QSEP Research Report, No. 354.

Weber, C. (2014), "Toward obtaining a consistent estimate of the elasticity of taxable income using difference-in-differences," Journal of Public Economics 117, 90-103. 